\title{
Burn injury
}

Marc G. Jeschke,2凶, Margriet E. van Baar ${ }^{3,4}$, Mashkoor A. Choudhry ${ }^{5}$, Kevin K. Chung 6 , Nicole S. Gibran ${ }^{7}$ and Sarvesh Logsetty ${ }^{8}$

Abstract | Burn injuries are under-appreciated injuries that are associated with substantial morbidity and mortality. Burn injuries, particularly severe burns, are accompanied by an immune and inflammatory response, metabolic changes and distributive shock that can be challenging to manage and can lead to multiple organ failure. Of great importance is that the injury affects not only the physical health, but also the mental health and quality of life of the patient. Accordingly, patients with burn injury cannot be considered recovered when the wounds have healed; instead, burn injury leads to long-term profound alterations that must be addressed to optimize quality of life. Burn care providers are, therefore, faced with a plethora of challenges including acute and critical care management, long-term care and rehabilitation. The aim of this Primer is not only to give an overview and update about burn care, but also to raise awareness of the ongoing challenges and stigmata associated with burn injuries.

Burn injuries are an under-appreciated trauma that can affect anyone, anytime and anywhere. The injuries can be caused by friction, cold, heat, radiation, chemical or electric sources, but the majority of burn injuries are caused by heat from hot liquids, solids or fire ${ }^{1}$. Although all burn injuries involve tissue destruction due to energy transfer, different causes can be associated with different physiological and pathophysiological responses. For example, a flame or hot grease can cause an immediate deep burn, whereas scald injuries (that is, from hot liquids or steam) tend to appear more superficial initially, due to rapid dilution of the source and energy. Alkaline chemicals cause colliquative necrosis (whereby the tissue is transformed into a liquid, viscous mass), whereas acidic burn causes a coagulation necrosis (whereby the architecture of the dead tissue can be preserved). Electrical injuries are entirely different because they can cause deep tissue damage that is greater than the visible skin injury; tissue damage in electrical injuries is correlated with the electric field strength (amperes and resistance of the tissue), although for ease of comprehension the voltage is often used to describe the circumstances of injury ${ }^{2}$. Thermal injury can also occur through cold. Frostbite is caused by a number of mechanisms including direct cellular injury from crystallization of water in tissue and indirect injury from ischaemia and reperfusion. These mechanisms lead not only to skin necrosis but also to deep tissue damage ${ }^{3}$. The particular cause of a burn injury determines the treatment approach. For example, although deep thermal burns are operated on immediately, the same approach would be an error in frostbite, in which the therapy of choice is moist rewarming, possible thrombolysis and watchful waiting.
In addition to determining the cause of a burn injury it is imperative to classify the injury according to its severity - its depth and size. Burns that affect the uppermost layer of the skin (epidermis only) are classed as superficial (first-degree) burns (FIG. 1); the skin becomes red and the pain experienced is limited in duration. Superficial partial-thickness (second-degree) burns (formerly known as 2A burns) are painful, weep, require dressing and wound care, and may scar, but do not require surgery. Deep partial-thickness (seconddegree) burns (formerly known as 2B burns) are less painful owing to partial destruction of the pain receptors, drier, require surgery and will scar. A full-thickness (third-degree) burn extends through the full dermis and is not typically painful owing to damage to the nerve endings, and requires protection from becoming infected and, unless very small, surgical management. Finally, a fourth-degree burn involves injury to deeper tissues, such as muscle or bone, is often blackened and frequently leads to loss of the burned part. Although superficial and superficial partial-thickness burns usually heal without surgical intervention, more severe burns need careful management, which includes topical antimicrobial dressings and/or surgery. Importantly, burns are classified as either minor or major. A minor burn is usually a burn that encompasses $<10 \%$ of the total body surface area (TBSA), with superficial burns predominating. By contrast, the burn size that constitutes a major burn is not commonly well-defined; some guidance to classify severe burn injuries are: $>10 \%$ TBSA in elderly patients, $>20 \%$ TBSA in adults and $>30 \%$ TBSA in children. Alongside injuries to the skin, burns can be accompanied by smoke inhalation or other physical trauma to other organs. 

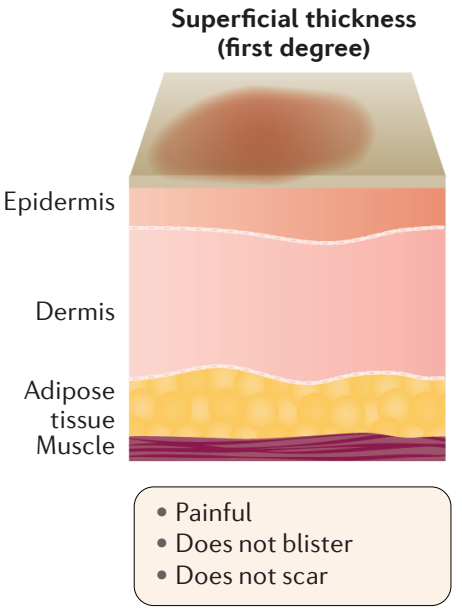

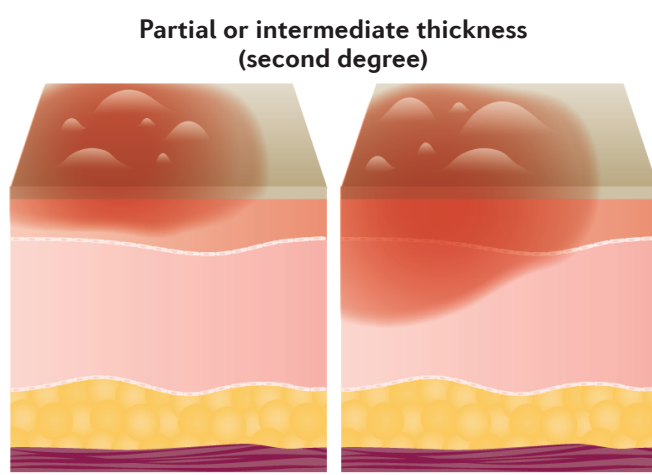

Superficial partial thickness burns do not require surgery, but may scar and be more painful

Blisters and weeps

- With increasing depth, increased risk of infection

- With increasing depth, increased risk of scarring

Deep partial thickness burns require surgery are less painful
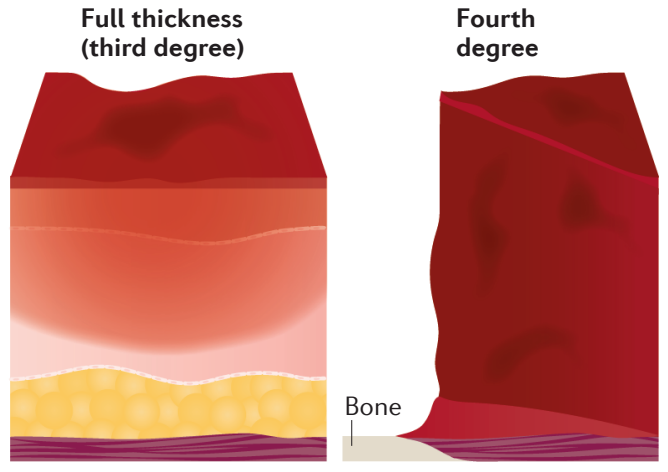
and form more scars and

Fig. 1 | Burn depth. Burn depth is an important factor in assessing patient care needs and, in particular, surgical needs; in general, the deeper the burn the more challenges there are to achieve good scar outcomes. First-degree (superficial thickness, affecting the epidermis only) burns are typically benign, very painful, heal without scarring and do not require surgery. Burns extending into the underlying skin layer (dermis) are classed as partial thickness or second-degree; these burns frequently form painful blisters. These burns range from superficial partial thickness, which are homogeneous, moist, hyperaemic and blanch, to deep partial thickness, which are less sensate, drier, may have a reticular pattern to the erythema and do not blanch. Third-degree (full thickness) and fourth-degree burns require surgery and, paradoxically, usually present with almost no pain.

The uniqueness of a severe or major burn injury is anchored in the body responses to it. After the injury, an immediate systemic and local stress response is triggered that, unlike sepsis or trauma, does not recover quickly. Severe burns cause a complex pattern of responses that can last up to several years after the initial insult ${ }^{4}$. In general, immediately after the insult, an inflammatory response is triggered to promote the healing process ${ }^{5,6}$. However, in severe burns, this inflammatory process can be extensive and become uncontrolled, leading to an augmented inflammation that does not induce healing but rather causes a generalized catabolic state and delayed healing. This response is almost unique to burns and is referred to as the hypermetabolic response; it is associated with catabolism, increased incidence of organ failure, infections and even death ${ }^{7}$.

\footnotetext{
Author addresses

${ }^{1}$ Ross Tilley Burn Center, Department of Surgery, Sunnybrook Health Science Center, Toronto, Ontario, Canada.

${ }^{2}$ Departments of Surgery and Immunology, University of Toronto, Toronto, Ontario, Canada.

${ }^{3}$ Association of Dutch Burn Centres, Maasstad Hospital, Rotterdam, Netherlands. ${ }^{4}$ Erasmus MC, University Medical Center Rotterdam, Department of Public Health, Rotterdam, Netherlands.

${ }^{5}$ Burn and Shock Trauma Research Institute, Alcohol Research Program, Stritch School of Medicine, Loyola University Chicago Health Sciences Division, Maywood, IL, USA. ${ }^{6}$ Department of Medicine, Uniformed Services University of the Health Sciences, Bethesda, MD, USA.

${ }^{7}$ Department of Surgery, University of Washington School of Medicine, Seattle, WA, USA. ${ }^{8}$ Departments of Surgery and Psychiatry, Rady Faculty of Health Sciences, University of Manitoba, Winnipeg, Manitoba, Canada.
}

Once the burn injury severity has been established, the patient needs to be appropriately referred and triaged. Care of a patient with major burn injury is resourceintensive, often takes place in a specialized centre ${ }^{8}$, and has a substantial impact not only on the life of the patient but also on the lives of caregivers and families ${ }^{9,10}$ — often for a long period of time. A recent study demonstrated that burn injuries affect morbidity and mortality for at least 5-10 years after the injury ${ }^{11}$. Thus, those involved in burn care must adapt their goals to move away from immediate survival as the main goal towards goals that address scarring, long-term well-being, mental health and quality of life. Indeed, the trauma care community recently adopted the goals of no death, no scar, no pain ${ }^{12}$.

In this Primer, we discuss key aspects of burn injury and provide an up to date description of the epidemiology and clinical care of patients with burn injuries. Mechanistically, we focus our discussion on severe burns, as these injuries exhibit profound inflammatory and metabolic effects that increase the risk of longterm sequelae and death, but principles of wound care and acute management can also be applied to minor burn injuries. The goals to reduce scarring and safeguard mental health are also described alongside other long-term outcomes of quality of life. We also discuss promising future therapies.

\section{Epidemiology}

Burn injuries result in lifelong physical and psychological scarring ${ }^{13}$, causing pain and influencing mental health, quality of life, ability to return to work and subsequent mortality ${ }^{10,11,14}$. Although information on burn 
epidemiology is essential for resource allocation and prevention, the available data are variable and inconsistent. The majority of data are from high-income countries and are directly related to access to health-care resources, differences in environments and the resources of the various health-care systems ${ }^{15-17}$. In lower income countries, fewer resources, geographical constraints and cost limit data collection and access to health care ${ }^{18}$. Additionally, cultural factors such as open-air cooking areas and loose clothing (for example, saris), domestic violence and dowry deaths contribute to regional variation ${ }^{19-21}$. To address the significant gap in information, the WHO is piloting an online, checkbox-based Global Burn Registry that aims to standardize reporting ${ }^{22}$.

Although burn injuries are decreasing in highincome countries, the prevalence of burn injuries remains high elsewhere, with $\sim 90 \%$ of burns occurring in low- and middle-income areas ${ }^{23,24}$. The WHO estimates that 11 million burn injuries of all types occur annually worldwide, 180,000 of which are fatal ${ }^{13}$. There is a wide variability in the incidence of burn injury ${ }^{23}$. For example, the number of burn-related deaths per 100,000 population ranges from 14.53 in Cote D'Ivoire to 0.02 in Malta $^{25}$. Burn-related deaths of children are 7 to 11 times higher in low-income than in high-income countries ${ }^{13,19}$.

In the USA, a bimodal age distribution of all burn injuries is evident, with the majority of injuries occurring in young children (1-15.9 years of age) and in those of working age (20-59 years of age $)^{1}$. Regardless of country, burns in children are more equally distributed between boys and girls, especially in toddlers ${ }^{1,24}$. However, this ratio changes as age increases; in most countries, nearly twice as many men are injured as women. An exception to this trend has been noted in Ghana and India, where up to three times more women are injured and die from burn injuries than men ${ }^{26,27}$.

The American Burn Association (ABA) National Burn Repository 2019 reports that, overall, flame burns are still the majority of injuries in the USA (41\%), with scalds second at $31 \%{ }^{1}$. Chemical $(3.5 \%)$ and electrical burn injuries (3.6\%) occur much less commonly ${ }^{1}$. Burns in children $<5$ years of age tend to be scald injuries, with increasing flame-related burns as age increases ${ }^{28}$. Around the world, burns in the elderly population are increasing, and are predominantly flame-related. However, scald injuries are increasing substantially as well $^{29}$. Finally, depending on the environment, burn injuries are more frequent in some vulnerable populations, such as those with epilepsy ${ }^{30}$.

\section{Mechanisms/pathophysiology}

Experimental and clinical studies have demonstrated that severe burns (regardless of the cause) result in the development of an extremely dysregulated inflammatory host response within a few hours of injury $y^{24,31-35}$. The inflammatory and stress responses are characterized by elevated levels of cytokines, chemokines and acute phase proteins as well as a hypermetabolic state that is driven by a sustained sympathetic tone that can persist beyond the acute phase of care ${ }^{5,36}$. A number of factors contribute to the magnitude of the host response: burn severity (percentage TBSA and burn depth); burn cause; concomitant inhalation injury; exposure to toxins; other traumatic injuries; and patient-related factors such as age, pre-existing chronic medical conditions, drug or alcohol intoxication, and timing of presentation to medical aid. Depending on the magnitude of the injury, the initial host response immediately after severe burn injury is similar to that after many other inflammatory conditions triggered by tissue destruction such as trauma or major surgery $^{33}$, which is helpful in initiating tissue repair and overall wound healing. However, after severe burns, the inflammatory cascade may be triggered multiple times during the course of clinical care after initial resuscitation, for example, during burn surgery or subsequent infectious complications. When the inflammatory cascade occurs repeatedly or remains uncontrolled, it can destroy host tissue and contribute to organ dysfunction and death. Although various pieces of the complex response after burn injury have been identified, how and in what sequence these pieces interact have not been resolved.

\section{Initial injury}

Immediately after injury, the burn wound can be divided into three zones: the zone of coagulation (with the most damage in the central portion); the zone of stasis or zone of ischaemia (characterized by decreased perfusion that is potentially salvageable); and the zone of hyperaemia (the outermost region of the wound characterized by increased inflammatory vasodilation). The degree of cellular injury varies depending on the zone of injury and spans the spectrum from immediate cellular autophagy within the first 24 hours following injury, delayed-onset apoptosis $\sim 24-48$ hours after the burn injury and the presence of reversible oxidative stress. The natural healing of these wounds involves dynamic and overlapping phases (FIG. 2) that include an inflammatory phase, which is initiated by neutrophils and monocytes homing to the injury site via localized vasodilation.

This inflammatory phase naturally serves to degrade necrotic tissue and initiate the cascade of signals required for wound repair. Following the inflammatory response, activation of keratinocytes and fibroblasts via various cytokines and growth factors helps usher in the proliferative phase that aims to restore vascular perfusion and further promote wound healing. The final phase of healing involves wound remodelling, in which collagen and elastin are deposited and continuously transform fibroblasts to myofibroblasts. Over time, a delicate balance between contraction of myofibroblast and re-epithelialization determines the quality and pliability of the repaired wound, and determines the extent of scar formation, which is characterized by fibrous malposition of collagen fibres ${ }^{36}$. We and others have hypothesized that optimal healing depends on an adequate 'pool' of cells derived from the bone marrow and on the balance between pro-inflammatory and anti-inflammatory mediators $^{37}$. In general, the complex healing response is targeted towards dermal and epidermal regeneration with the goal of restoring closure of the skin barrier as well as pliability and functionality of the skin. However, wounds can heal with abnormal scars that are characteristically active, red, itchy, painful and disfiguring - termed hypertrophic or keloid scars (see below). 


\section{Shock}

Alongside cytokines and other inflammatory mediators at the site of injury, stress hormones such as catecholamines and cortisones are released by the adrenal glands, all of which have systemic effects. Burn injury usually results in a distributive shock ${ }^{38}$, an abnormal physiological state in which tissue perfusion and oxygen delivery is severely compromised owing to marked capillary leakage of fluid from the intravascular to interstitial space, that contributes to profound tissue oedema and

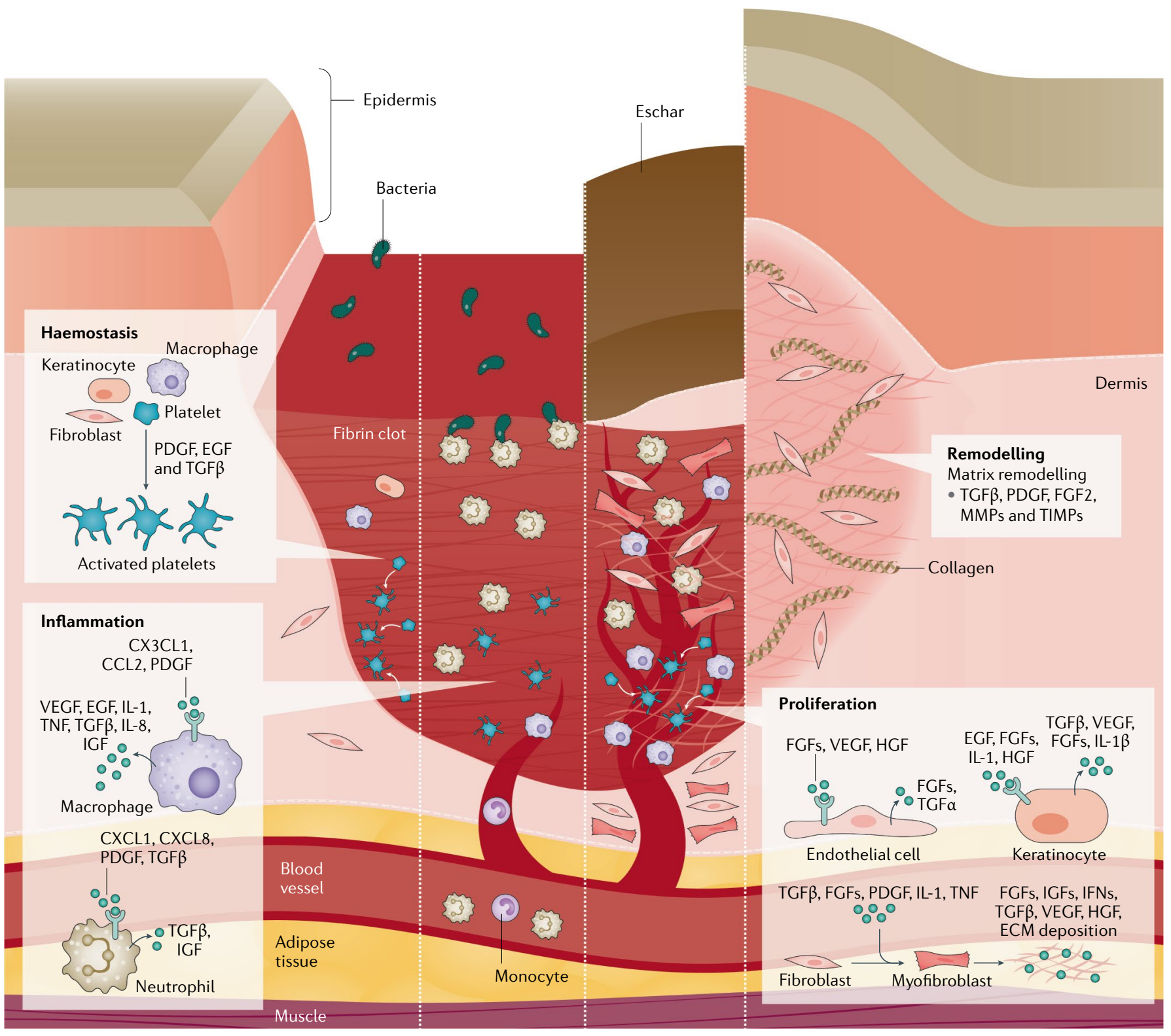

Fig. 2 | Four phases of natural wound healing. Haemostasis occurs immediately after the injury and involves vasoconstriction, platelet activation and aggregation, and release of clotting and growth factors (such as platelet-derived growth factor (PDGF), epidermal growth factor (EGF) and transforming growth factor- $\beta$ (TGF $\beta$ )) by platelets, keratinocytes, macrophages and fibroblasts, resulting in fibrin clot deposition at the injury site, which serves as a provisional matrix for subsequent stages of healing. Monocytes (and macrophages) and neutrophils are recruited to the injury site owing to localized vasodilation and initiate the inflammation phase. Inflammation begins within 24 hours of the injury and lasts for weeks to months depending on the severity of injury. Neutrophils and macrophages release cytokines and chemokines (including IL-1, IL-8 and tumour necrosis factor (TNF)) and growth factors (including TGF $\beta$, insulin-like growth factor (IGF) and vascular endothelial growth factor (VEGF)), and remove debris and pathogens from the injury site. The next phase, proliferation, involves the recruitment and activation of fibroblasts and keratinocytes to the wound site. Proliferation is characterized by replacement of the provisional matrix with a connective tissue matrix, granulation (new connective tissue and microscopic blood vessels), angiogenesis and epithelialization. Keratinocytes assist in both epithelialization (wound surface closure) and angiogenesis (restoration of blood flow), which are vital to wound healing. Endothelial cells are activated by growth factors (VEGF, hepatocyte growth factor (HGF) and fibroblast growth factors (FGFs)) to initiate angiogenesis. Resident fibroblasts are transformed to myofibroblasts, which are involved in extracellular matrix (ECM) deposition. In the final phase, remodelling, granulation tissue matures and the ECM is remodelled under the influence of growth factors, matrix metalloproteinases (MMPs) and tissue inhibitors of metalloproteinases (TIMPs), which leads to increased tensile strength. The length of healing depends on multiple factors including the injury severity, inflammatory cascade activation and nutrition. IFN, interferon. 
fluid accumulation ${ }^{39,40}$. The marked capillary leakage can be attributed to oxidative stress that is characterized by increases in the levels of nitric oxide and inflammatory mediators, which damages the vascular endothelium. Burn injury also depresses cardiac function within a few hours of injury, lasting 24-48 hours, via oxidative stress, the release of inflammatory mediators (such as IL-6 and tumour necrosis factor (TNF)) and cellular alterations (such as apoptosis and necrosis) ${ }^{32,34,39,41}$. The decrease in cardiac function and relative hypovolaemia, along with low blood flow caused by vasoconstriction, affects perfusion of tissues and organs (that is, distributive shock), including the lungs, liver and gastrointestinal tract - augmenting tissue and organ dysfunction and damage. The state of shock continues even if hypovolaemia is corrected ${ }^{38}$. Furthermore, the cardiovascular dysfunction can further exacerbate the systemic inflammatory response into a vicious cycle of accelerating organ dysfunction (summarized in REF. ${ }^{42}$ ).

\section{Hypermetabolic state}

After an initial ( 72-96 hours) hypometabolism (ebb phase $)^{43}-$ which is potentially caused by intracellular processes, e.g. increased endoplasmic reticulum stress and mitochondrial dysfunction ${ }^{44}$ and characterized by decreased metabolic rate and intravascular volume, poor tissue perfusion and low cardiac output - a hypermetabolic state is typically observed after injury in patients with severe burns (flow phase) $)^{45,46}$. The hypermetabolic state after burn injury persists for up to 36 months after the initial insult ${ }^{4}$. Stress hormones such as catecholamines, glucocorticoids (produced by the adrenal glands) and glucagon (produced by the pancreas) increase blood pressure, peripheral insulin resistance and breakdown of glycogen, proteins and lipids. The results of these effects are increased resting energy expenditure, increased body temperature, total body protein loss, muscle wasting and increased stimulated synthesis of acute-phase proteins (such as insulin-like growth factor 1 (IGF-1), which has anabolic effects), ultimately resulting in organ catabolism associated with organ dysfunction and death ${ }^{4,47-49}$.

Although these responses are reported in patients with other types of trauma or critical illness, their magnitude and duration in patients with severe burn injury is of consequence. Sustained release of catecholamines, glucocorticoids, glucagon and dopamine (released from the brain) seem to initiate a cascade of events leading to an acute hypermetabolic response and an ensuing catabolic state (FIG. 3). The mechanism(s) underlying this complex response remain to be established, but studies have suggested that the continuous and sustained release of pro-inflammatory mediators such as cytokines, chemokines and acute phase proteins (namely, IL-1, IL-6, TNF and IGF-1) ${ }^{4}$ may further contribute to the hypermetabolic state in patients with moderate to severe burn injury. Regardless of the underlying mechanistic detail, an effective treatment to abolish or reduce the hypermetabolic response is not available. However, the recent discovery of the browning of the white adipose tissue due to inflammation or upregulation of mitochondrial brown fat uncoupling protein 1 (which is responsible for thermogenic respiration) after burn injury ${ }^{47,50}$ seems to induce metabolic and immune changes that further augment hypermetabolism and immune dysfunction - an avenue that may lead to new treatment options.

\section{Immune dysregulation and infection}

In addition to hypovolaemic and hypermetabolic responses, burn injury has a profound effect on the immune system $^{34,35,51,52}$. Immune cells, including monocytes, macrophages and neutrophils, which are activated in response to burn injury within a few hours, recognize endogenous factors such as damage-associated molecular patterns (DAMPs) or alarmins that are generated as a result of burn-mediated tissue damage. DAMPs and their exogenous counterparts, pathogen-associated molecular pattern molecules (PAMPs), are recognized via pattern recognition receptors, namely Toll-like receptors (TLRs) and NOD-like receptors (NLRs). The ligation of TLRs and NLRs by their specific ligands results in the activation of downstream inflammatory pathways, leading to activation of NF- $\kappa \mathrm{B}$, a master transcription factor involved in the release of multiple inflammatory mediators (such as IL-1, IL-6, IL-8, IL-18 and TNF). The release of these cytokines and chemokines furthers the cycle of inflammation leading to systemic inflammatory response syndrome (in which uncontrolled cytokine release leads to excessive leukocyte recruitment, fever or hypothermia, tachycardia and tachypnoea $)^{53}$. Interestingly, other immune functions are severely compromised, including macrophage antigen presentation or neutrophil killing of invading pathogen ${ }^{54-56}$. Moreover, T cell proliferation and IL-2 production are also suppressed ${ }^{57-62}$. Together, these events lead to the development of a compromised adaptive immune response, resulting in enhanced susceptibility to infection (FIG. 4).

Indeed, patients with severe burn injuries are at higher risk of developing infectious complications. Ventilator-associated pneumonia is also a common finding in patients with severe burn injuries ${ }^{63}$. Additional sources for infection in these patients can be their own microbiota associated with skin, respiratory tract and intestines. Compromised host defence from the disrupted skin barrier in patients with burn injury leads to increased susceptibility to infection (predominantly bacterial, but also yeast, fungal and viral), increase virulence from specific pathogenic organisms and the subsequent development of organ failure ${ }^{33,59,64}$. Indeed, in a recent autopsy study, $>60 \%$ of deaths in patients with burn injury were attributable to infectious complications and nearly all had at least one associated organ failure, with many having multiple organ failure ${ }^{65-67}$. Each episode of sepsis (BOX 1) can lead to organ dysfunction, which eventually leads to organ failure that affects different organs, including the kidneys, lungs, liver, gastrointestinal tract, heart and bone marrow.

Various studies support the suggestion that the gut, being the major source of bacteria and bacterial products, also plays an important part in pathogenesis after burn injury ${ }^{68,69}$. Previous studies have shown an increase in intestinal bacterial growth after burn injury, resulting from diminished gut immunity, hypoperfusion and gut dysmotility ${ }^{70}$. Furthermore, an increase in intestinal 


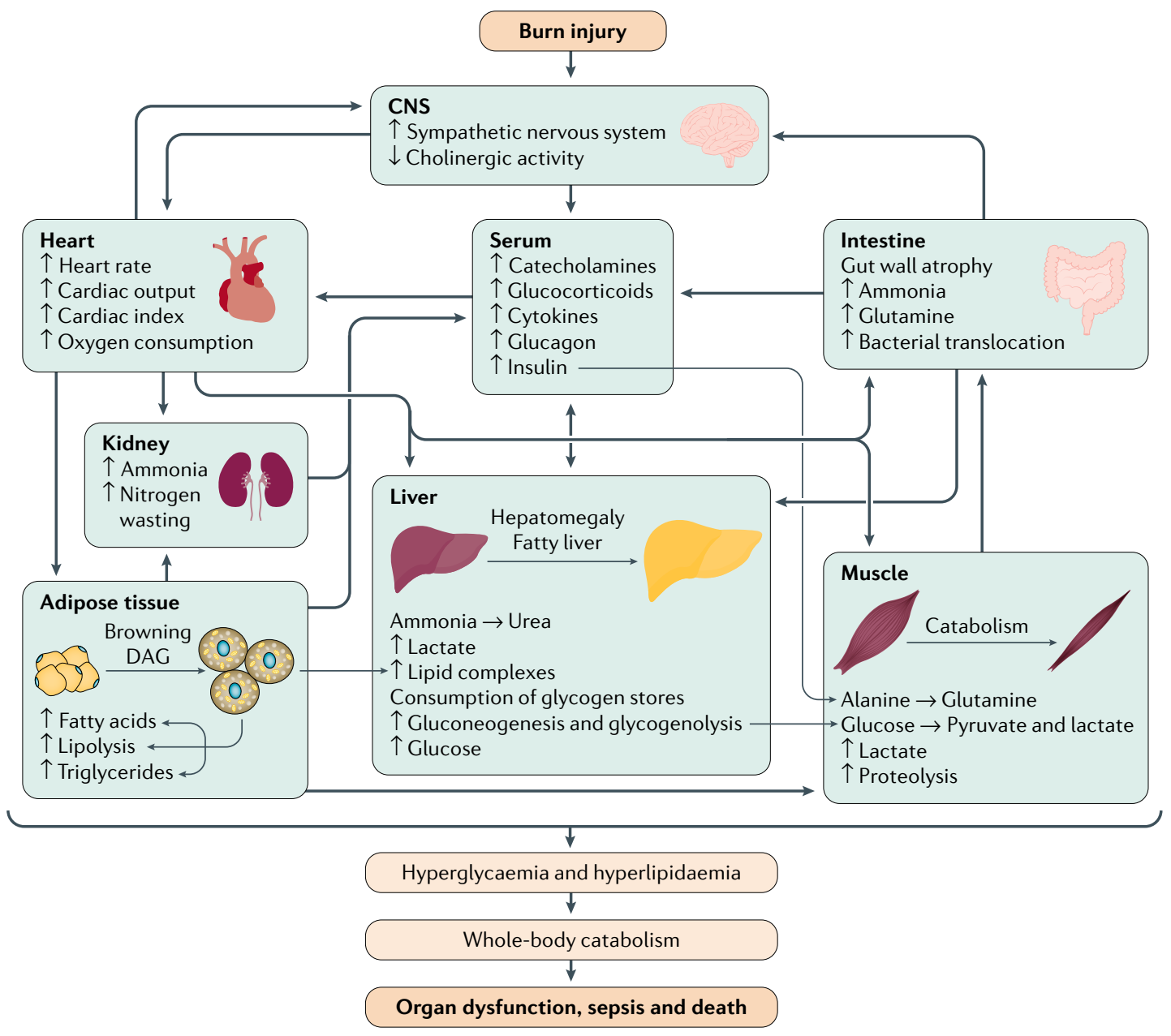

Fig. 3 | Hypermetabolic state in burn injury. Severe burn injury induces a unique and remarkably complex response that involves the release of stress hormones and pro-inflammatory mediators. The immediate response leads to a hypometabolic response that lasts for $\sim 72-96$ hours (ebb phase), but then rapidly turns into the flow phase that can persist for years after the initial injury. Stress mediators, such as catecholamines, glucocorticoids and cytokines, are released into the system and cause a plethora of systemic responses. The heart goes into a hyperdynamic overdrive, increasing circulation and blood flow to increase oxygen and nutrient delivery. However, increased stress signalling causes changes in organ function and metabolic demand. Protein is degraded to deliver energy for hepatic function, the gut develops mucosal atrophy to absorb more nutrients but also enabling bacterial translocation. The kidneys are hyperperfused but oxygen delivery is decreased, leading to acute kidney injury and stress signals from the kidney. The interplay between these organs accumulates, leading to metabolic and inflammatory overdrive that subsequently causes white adipose tissue to change to brown adipose tissue. Brown adipose tissue releases energy and induces substantial lipolysis with the accompanying expression of lipotoxic intermediates, such as triglycerides, free fatty acids and diacylglycerols (DAG), all of which are transferred to the liver. The liver is unable to metabolize all of the accumulating substances and develops hepatomegaly. In turn, hyperlipidaemia and hyperglycaemia with insulin resistance is present, which worsens the hypermetabolic and inflammatory state. If hypermetabolism cannot be diminished or decreased, holistic catabolism ensues and, subsequently, multiple organ failure and death. CNS, central nervous system.

permeability (due to hypoperfusion and subsequent tissue inflammation and damage) has also been documented in patients and in animals within a few hours of burn injury ${ }^{70-72}$ and enables the gut bacteria to enter extra-intestinal sites such as mesenteric lymph nodes, liver and lungs. Indeed, increased bacterial translocation has been noted within the first few days after burn injury ${ }^{70-72}$. However, this process becomes recurrent when the burn injury is followed by additional triggers that lead to gut hypoperfusion, such as peri-operative haemorrhagic shock and infectious complications. The microbial communities in faecal samples from patients with burn injury have been shown to be different from those observed in faecal samples from healthy controls $^{73,74}$. Specifically, the faecal microbial communities of controls were dominated by the Bacteroidaceae, Lachnospiraceae, and Ruminococcaceae families. The faecal samples from patients with burn injuries exhibited a marked decrease in the relative abundance of these three families, but also demonstrated sharp increases in the relative abundance of the Enterobacteriaceae, a finding that has been replicated in mice ${ }^{74}$. Furthermore, results obtained using in situ hybridization suggest large populations of Enterobacteriaceae in close proximity to 


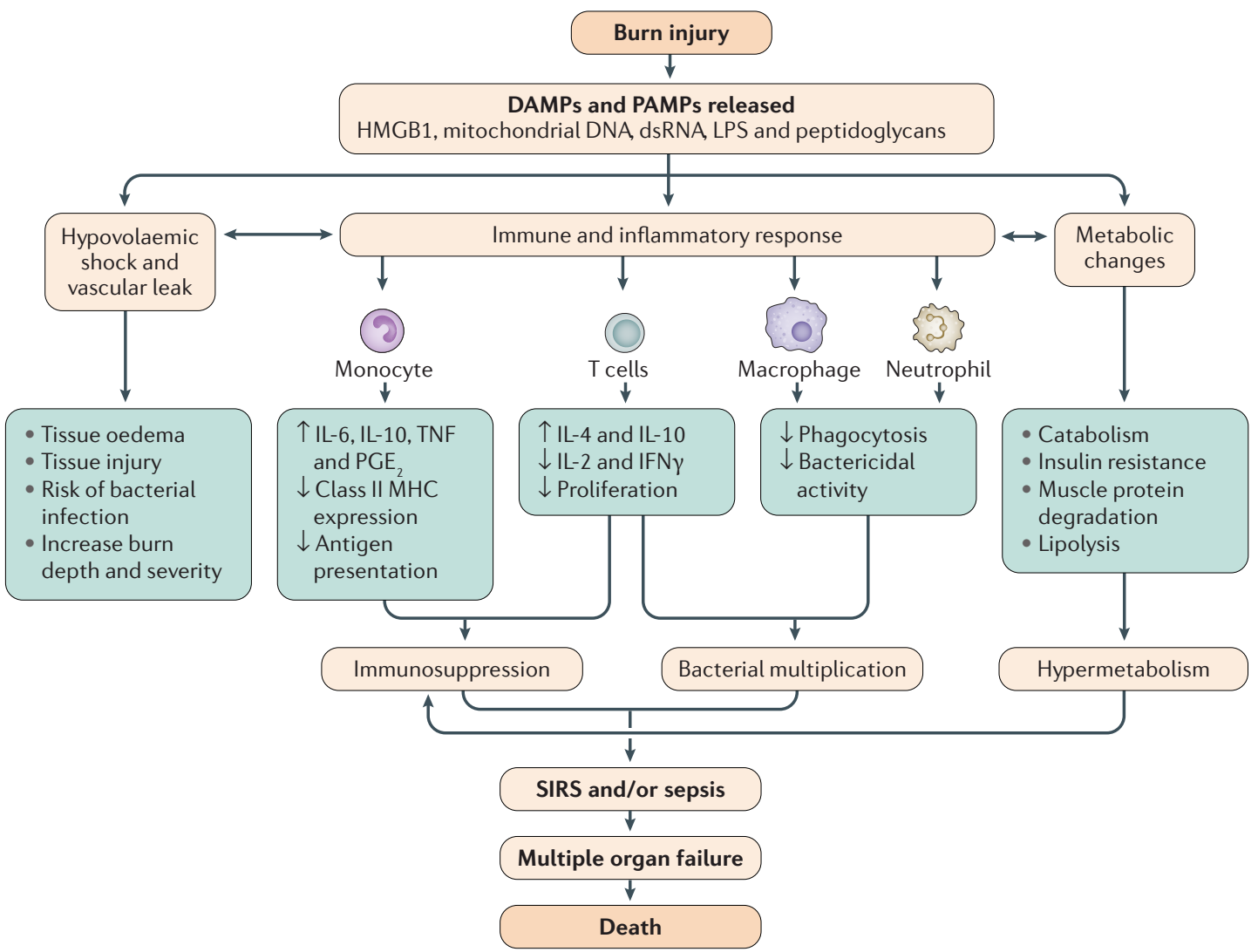

Fig. 4 | Events leading to sepsis and multiple organ failure following burn injury. Tissue injury following severe burns results in release of endogenous damage-associated molecular patterns (DAMPs) such as mitochondrial DNA ${ }^{263}$ and double-stranded RNA (dsRNA), which along with exogenous pathogen-associated molecular pattern molecules (PAMPs) such as lipopolysaccharides (LPS) and peptidoglycans, can induce vascular leak, an inflammatory response and metabolic changes. Vascular leak and transfer of intravascular fluid to third spaces leads to tissue oedema and further injury. The inflammatory response can result in immunosuppression and ineffective response to bacterial invasion. Metabolic changes include increased muscle protein degradation, insulin resistance and increased cardiac load. The culmination of these events is often systemic inflammatory response syndrome (SIRS), an inflammatory state affecting the whole body, which can lead to multiple organ failure, and ultimately, death. MHC, major histocompatibility complex; $\mathrm{PGE}_{2}$, prostaglandin $\mathrm{E}_{2}$; TNF, tumour necrosis factor. Adapted from REF. ${ }^{264}$, Springer Nature Limited.

the villi in the small intestine of mice receiving burn injury ${ }^{74}$. This observation provides further evidence that intestinal bacteria and their products (such as endotoxin) can cross the intestinal epithelial barrier into the systemic or lymphatic circulation and contribute to the pathology after burn injury. Changes in the microbial community or diversity have been implicated in the development of many diseases including allergies, obesity, inflammatory bowel disease and many infectious diseases ${ }^{75-80}$. However, a more systematic approach is needed to better appreciate the role of gut-derived bacteria or the microbiome in pathogenesis after burn injury.

\section{Other injuries}

Burn injuries can also lead to alterations and injuries in other organs. A common injury occurring with burns is inhalation injury (respiratory tract or lung tissue injury), caused by heat or inhalation of smoke or chemical products of combustion and leading to various degrees of damage (BOX 2). Usually, inhalation injury is present in conjunction with the burn and can range from a minor injury to a very severe and necrotic injury pattern.
Inhalation injury increases the risk of pulmonary complications, including ventilator-associated pneumonia, fluid requirements and mortality ${ }^{81}$. However, measurement of the severity of inhalation injury is currently not well defined, despite some efforts and an existing grading system based on bronchoscopy ${ }^{82}$, making assessment of the true effect of inhalation injury challenging.

A small percentage of burn injuries are associated with concomitant trauma, which commonly includes traumatic brain injury, injury to the abdominal or thoracic cavities, fractures or complex soft tissue injury (such as crush injury or degloving injury, with multiple layers of tissue involved). In general, patients with burns and traumatic injuries have worse outcomes than those without trauma ${ }^{83}$. A comprehensive assessment of patients by trauma and burn teams needs to be conducted to optimize outcomes in these patients with complex and challenging injuries.

Additionally, burn injury affects all organs to some degree, owing to the systemic response to the burn. Possible effects include brain atrophy, pulmonary damage leading to pneumonia and/or acute respiratory 


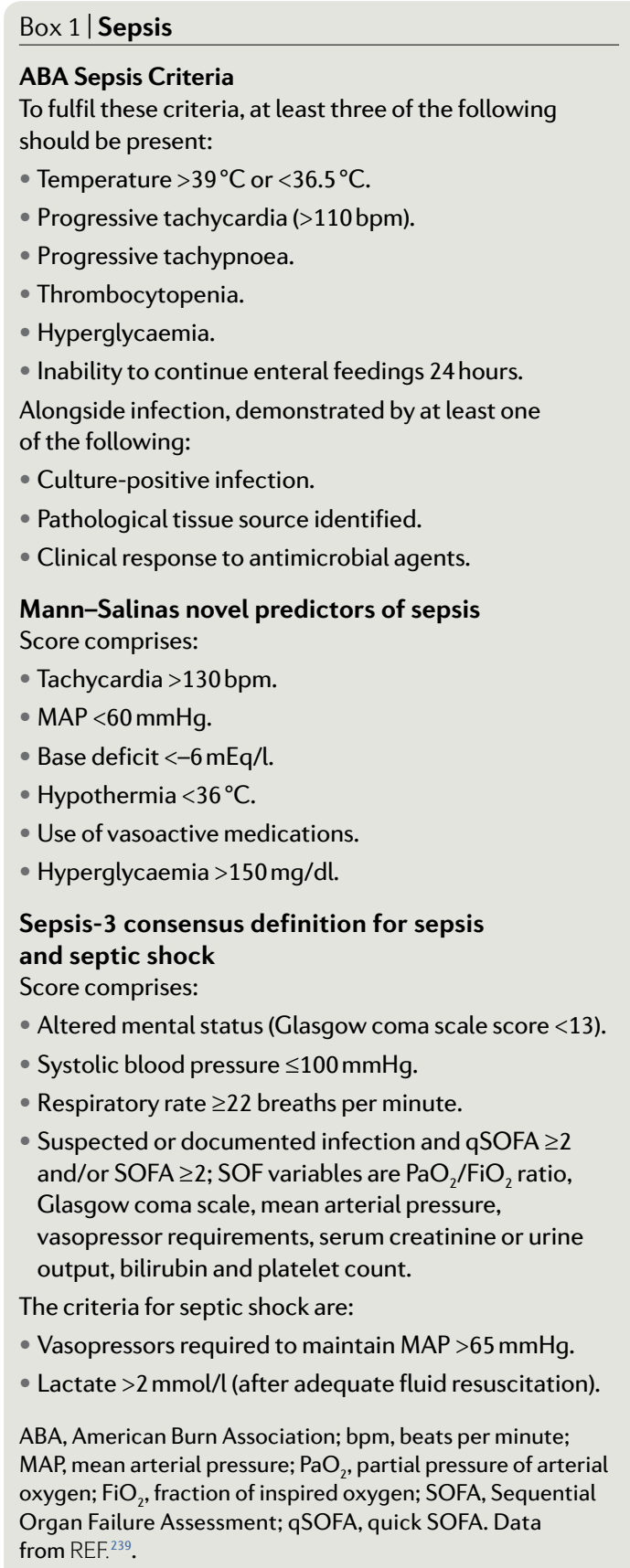

distress syndrome (ARDS), acute renal failure, liver failure, hepatic fatty infiltration, gut atrophy, lipolysis and fat catabolism, cardiac dysfunction, and thymus and immune dysfunction and depletion. These effects lead to immunocompromise, loss of bone mineral density, hormone depletion and dysfunction, and thyroid dysfunction, all of which are common in the complex picture of burn-associated hypermetabolism. In an autopsy study, for example, multiple organ failure was a primary cause of $>70 \%$ of all burn-related deaths ${ }^{84}$.

Although very rare, a potential complication that has been documented in patients with burn injuries is irritation of the eye ${ }^{85}$. Exposure to hot air, steam or flames can burn the face and eyes or eyelids, which can affect the normal function of the eye. Furthermore, injury to the eyelid can compromise the barrier and predispose the burn wound to foreign bodies and infection.

\section{Diagnosis, screening and prevention Prevention}

Burn injuries result in permanent scarring and adverse long-term sequelae that affect burn survivors and their caregivers ${ }^{9,10,14}$. Unfortunately, as vulnerable populations are disproportionately injured, the burden from these injuries further marginalizes already disadvantaged groups $^{18}$. A central tenet of modern burn care is that the majority of burn injuries are preventable ${ }^{13,86}$. As a result, the ABA encourages participation in prevention programmes as part of burn centre verification, and the WHO has proposed a plan for burn prevention and care at a global level ${ }^{87,88}$. To encourage prevention implementation globally, the WHO published a collection of successful strategies from high-, middle- and low-income countries. These strategies range from grassroots changes of lifestyle (for example, safe wood burning stoves) to legislative regulation (for example, on children's sleepwear flammability $)^{89}$.

Alongside legislative strategies, successful implementation of prevention programmes (for example, the introduction of fire-safe cigarettes and reduced flammability of children's clothing) has reduced the incidence of burn injuries in high-income countries ${ }^{90-93}$. However, these efforts may not be as effective in low- and middleincome countries. In these settings, local social factors must be taken into consideration, for example, most low-income countries do not have water heaters or the resources for smoke detectors in each home. The Haddon Matrix (assessing pre-event, event-related and post-event factors) should be considered when constructing prevention programmes ${ }^{89,94}$ as the matrix helps to organize interventions for appropriate upstream, midstream and downstream targets. This approach can be applied at a regional level or even at a case level ${ }^{95,96}$. FIGURE 5 provides an example of a Haddon Matrix under development by the authors (S.L.) to guide a programme for prevention of paediatric burn injuries.

To be successful, regional burn prevention programmes should concentrate on factors that influence the incidence of burn injury in the local environment, knowledge dissemination strategies and data collection methods to identify the areas of focus and evaluate programme effectiveness ${ }^{89}$. For example, in some countries, factors such as loose clothing and cooking on open fires are important areas of focus. In other regions, the occurrence of electrical burns has increased substantially as industrialization has taken place ${ }^{21}$. Prevention strategies can also be applied at a more personal level. For example, a review of injuries sustained by firefighters identified that burns occurred in common patterns associated with gaps in equipment, which can guide future equipment modifications and improvements ${ }^{97}$. Development of prevention programmes must also take into consideration knowledge access and engagement with the selected media. For example, strategies to 'gamify' knowledge acquisition using mobile phone applications could reach a wider audience than a television commercial in some regions and for certain age groups ${ }^{98}$. 


\section{Box 2 | Inhalation injury}

Inhalation injury can be divided into three types: systemic toxicity due to products of combustion (carbon monoxide ( $\mathrm{CO}$ ) and cyanide poisoning); upper airway thermal injury; and lower (bronchi and distal) airway chemical injury. Patients can sustain all of these in a closed-space fire. CO poisoning, more accurately categorized as a systemic intoxication, is easily diagnosed from the serum carboxyhaemoglobin level determined as part of the arterial blood gas measurement at hospital admission. Administration of $100 \%$ oxygen reduces the carboxyhaemoglobin half-life from 4 hours on room air $\left(21 \% \mathrm{O}_{2}\right)$ to $\sim 45$ minutes; patients with $\mathrm{CO}$ poisoning can have normal carboxyhaemoglobin levels on arrival at the hospital. Even when early intubation is not indicated, $100 \% \mathrm{O}_{2}$ administration should be continued until carboxyhaemoglobin levels are confirmed to be $<5 \%$. Although proponents of hyperbaric oxygen (HBO) therapy - in which pure oxygen is delivered in a pressurized room or tube - have argued that the treatment reduces long-term neurological sequelae of $\mathrm{CO}$ poisoning, prospective randomized trials have presented conflicting data regarding long-term cognitive function ${ }^{249}$. Cyanide toxicity is often clinically diagnosed by persistent acidosis without another systemic source; empirical treatment with cyanocobalamin has increased despite limited evidence for its safety and effectiveness. Upper airway burns can be diagnosed by assessing symptoms of hoarseness, stridor or oropharyngeal soot and examining the posterior pharynx for oedema or mucosal slough. Injuries to the lower airway tend to be due to chemical pneumonitis from products of combustion. Thermoregulation by the oropharynx is sufficient to cool most gases before they reach the distal lung parenchyma; however, steam is less efficiently cooled and may cause a thermal injury to the lower airways. Lower airway burns can be diagnosed with fibre optic bronchoscopy or by evidence of small airway inflammation and obstruction on CT. Prolonged mechanical ventilation complicates the care of patients with burn injuries, with or without inhalation injury. Patients with inhalation injury are at risk of developing acute respiratory distress syndrome (ARDS) from direct airway injury and their increased fluid resuscitation requirements from the inflammatory response to the burn injury. Many burn centres have adopted the use of lower tidal volumes and reduced airway plateau pressures to treat ARDS based on compelling data from the ARDSnet ${ }^{250}$. The applicability of extracorporeal membrane oxygenation in patients with inhalation injury must also be determined.

Literacy patterns, cultural appropriateness (which is particularly challenging to address in countries with more than one official language and no clear common language) and access to the recommended technologies can be barriers to this approach; for example, it is not possible to avoid open fire cooking until a cheap, safe, readily available alternative is identified ${ }^{95,98,99}$. Furthermore, correction of one risk factor might introduce another; for example, use of liquefied petroleum gas burners as an alternative to open fire cooking without proper training can result in more injuries ${ }^{100}$. Robust data collection and reporting and evidence-based decision making is needed to provide the information to drive this process. Although this information is more readily available in high-income countries, the countries with the highest rates of burn injury may not currently have the infrastructure to collect these data ${ }^{89}$. To address this need, the WHO has started the Global Burn Registry to aid data collection in low-resource settings and address this knowledge gap ${ }^{22}$.

\section{Diagnosis}

Accurate assessment of the severity of a burn injury is paramount because it forms the basis for all subsequent treatment decisions, triage plans and assessment of medical futility. Whenever possible, decisions about how to proceed after diagnosis and screening should incorporate patient preferences and expectations about quality of life $\mathrm{e}^{101}$. Optimal assessment of the severity of burn injury must involve a systematic methodical approach, such as that described in course materials for the Advanced Trauma Life Support ${ }^{102}$ (ATLS) by the American College of Surgeons Committee on Trauma, Emergency Management of the Severe Burn ${ }^{103}$ (EMSB) by the Australian and New Zealand Burn Association, and Advanced Burn Life Support ${ }^{104}$ (ABLS) by the ABA. Such diagnostic approaches must involve an orderly sequential evaluation (phase I of management), including the primary and secondary survey, that considers the need for further consultation and possible transport to optimize outcomes at specialized centres ${ }^{105}$.

Primary survey. The primary survey at the scene of the injury or in the emergency department comprises immediate standardized assessment - in this order — of: the airway, breathing, ventilation, circulation and cardiac status, disability, neurological deficit, gross deformity and degree of exposure (requiring complete disrobing to enable identification of associated injuries). To avoid hypothermia, especially in children and the elderly, this assessment must be conducted while maintaining a warm environment ${ }^{106}$. A preliminary estimate of the size of the burn using Lund and Browder diagrams ${ }^{107}$ for children and, for adults, the Rule of Nines ${ }^{108}$ (FIG. 6) is essential at this time because the amount of oral or intravenous fluid resuscitation is based on the burn size (percentage TBSA). Given the life-threatening nature of respiratory failure, assessing burn severity must also include early determination of whether the patient has a smoke inhalation injury (BOX 2). Exposure to products of combustion in a closed space, facial burns and soot in the oral cavity do not in themselves indicate an inhalation injury but strongly necessitate further physical examination of the posterior pharynx for evidence of thermal injury, including mucosal erythema, sloughing and swelling or soot in the vocal cords. Clinical signs such as stridor, hoarseness, carbonaceous sputum and dyspnoea also suggest inhalation injury and warrant further work-up ${ }^{109}$. However, even in resource-rich settings, in the majority of patients, diagnosis is made primarily on clinical appearance.

Secondary survey. The secondary survey, often in the emergency department or the burn centre, includes laboratory analyses and imaging, as indicated by evidence of other trauma or comorbidities. The secondary survey includes ensuring adequate tetanus prophylaxis, as burns are open wounds. The initial laboratory analyses in patients with burn a size of $\geq 15 \%$ TBSA include complete blood count, electrolyte assessment, coagulation profile and arterial blood gas measurement. In patients with suspected smoke inhalation injury, normal oxygenation and chest radiographs do not rule out the diagnosis as the pulmonary inflammatory response may take time to develop ${ }^{110,111}$.

The secondary survey importantly includes a definitive evaluation of the burn severity including depth (FIG. 1) and size based on TBSA (FIG. 6). Understanding which burn wounds will heal and which will benefit from early excision and grafting can be challenging, owing to a lack of widely accepted validated noninvasive imaging techniques to estimate burn depth. Furthermore, 


\begin{tabular}{|c|c|c|c|c|}
\hline & Host and human factors & Object or substance & Physical environment & Sociocultural environment \\
\hline $\begin{array}{l}\text { Pre-event } \\
\text { (before } \\
\text { injury) }\end{array}$ & $\begin{array}{l}\text { - Teach burn safety } \\
\text { - Teach bath water } \\
\text { temperature testing } \\
\text { - Supervision in baths } \\
\text { - Lack of experience } \\
\text { in young mothers }\end{array}$ & $\begin{array}{l}\text { - Consider water } \\
\text { heater temperature } \\
\text { regulators } \\
\text { - Anchor stoves and } \\
\text { ovens to walls so } \\
\text { they do not tip over }\end{array}$ & $\begin{array}{l}\text { - Address } \\
\text { environmental factors } \\
\text { - Test water } \\
\text { temperature } \\
\text { - Use of passive } \\
\text { temperature } \\
\text { governing valves }\end{array}$ & $\begin{array}{l}\text { Identify social } \\
\text { determinants of health } \\
\text { to guide who and where } \\
\text { to target prevention } \\
\text { strategies }\end{array}$ \\
\hline $\begin{array}{l}\text { Event } \\
\text { (during } \\
\text { injury) }\end{array}$ & $\begin{array}{l}\text { - Minimize exposure } \\
\text { - Prevent further } \\
\text { contamination }\end{array}$ & $\begin{array}{l}\text { - Remove from } \\
\text { additional risks } \\
\text { - Assess for other } \\
\text { injuries }\end{array}$ & $\begin{array}{l}\text { - Keep child } \\
\text { comfortable } \\
\text { - Prevent hypothermia } \\
\text { - Contact } \\
\text { emergency care }\end{array}$ & $\begin{array}{l}\text { - Nearest health-care } \\
\text { facility } \\
\text { - Social supports }\end{array}$ \\
\hline $\begin{array}{l}\text { Post-event } \\
\text { (after } \\
\text { injury) }\end{array}$ & $\begin{array}{l}\text { - Rehabilitation } \\
\text { - School reintegration }\end{array}$ & $\begin{array}{l}\text { - Counselling for } \\
\text { potential risk } \\
\text { avoidance } \\
\text { behaviour }\end{array}$ & $\begin{array}{l}\text { Review of home } \\
\text { environment for } \\
\text { potential safety } \\
\text { improvements }\end{array}$ & $\begin{array}{l}\text { - Supply target at-risk } \\
\text { groups with specific } \\
\text { prevention tools } \\
\text { (for example, education } \\
\text { about keeping coffee } \\
\text { pot wires out of reach } \\
\text { of toddlers) }\end{array}$ \\
\hline
\end{tabular}

Fig. 5 | The Haddon Matrix for burn prevention. The Haddon Matrix is a means of understanding traumatic injury that examines aetiological relationships instead of using descriptive terms ${ }^{18}$. This approach is essential to transition from the concept of burn injury as an 'accident' that is unpreventable, to the concept of injury being related to factors that can be modified. For example, we shift the concept from a child 'accidentally' turning on the hot water in a bathtub, to an unsupervised (change supervision) child turning on the hot water (control the water temperature at the boiler, or with a mixing valve). In using this approach, it is important not to assign blame, but to recognize the resources needed or factors that influence the cause of the traumatic event. For example, by examining the reasons why the child was unsupervised (parental mental health, other young siblings in the family, overstretched parents) and how to control the hot water (turn down the water heater, child-proof lockable tap, preset water temperature at the tap), programmes can be established to address them.

estimates of burn size and depth made before admission to a burn centre - and even by experts - have consistently been shown to be inaccurate in spite of standardization attempts and the availability of tools such as the Lund and Browder diagrams ${ }^{107}$ and the Rule of Nines ${ }^{108}$. Given the unreliability of burn size and depth assessment by clinicians who are not burn experts and the expense and logistics of transferring patients to higher levels of care, development of creative technological solutions for consultations is essential. New approaches in development include the use of computer-assisted programs to improve burn size estimation and enable focused and accurate telemedical consultation ${ }^{112,113}$. Telemedicine programs encompassing either real-time video conferencing or store-forward pictures of the wounds provide an option for burn experts to facilitate triage by assessing injury severity prior to transfer. Precise burn depth assessment can be challenging especially in patients at extremes of age and with thinner skin. To address this issue, several innovative devices incorporating laser Doppler imaging ${ }^{114,115}$, harmonic ultrasound imaging ${ }^{116}$, optical coherence tomography ${ }^{117}$ and high-resolution infrared thermography ${ }^{118}$ have been developed and introduced into preclinical and limited clinical trials. Validation of these modalities requires both comparison of digital images with histological specimens of burned skin as well as objective correlation of the data with burn depth assessments by clinical experts. Unfortunately, the expense of integrating these into daily practice has presented a barrier to widespread use.
A critical aspect of the secondary survey is to calculate the initial fluid infusion rate needed. Historically, resuscitation following burn injury has centred on a variety of established formulae that estimate total 24-hour crystalloid-based fluid requirements. Using these formulae, the ABA guidelines recommend the estimation of 24-hour fluid volume using $2-4 \mathrm{ml} / \mathrm{kg}$ per \%TBSA burned $^{119}$. In practice, these formulae are primarily used to calculate the initial fluid infusion rate that should be initiated in the early phases of resuscitation after a severe burn injury, after which there is immediate divergence from the hourly fluid infusion rate estimates. This divergence stems from the fact that fluids are titrated to a target urine output $(0.3-0.5 \mathrm{ml} / \mathrm{kg}$ per hour) rather than remaining fixed as the traditional formulae estimate. Hence, these formulae are used only to derive the initial fluid infusion rate. More recently, the Rule of Tens has been introduced to simplify the estimation of the initial fluid infusion rate and comprises three steps. First, the burn size (percentage TBSA) is estimated to the nearest 10 . Second, the percentage TBSA is multiplied by 10 to compute the initial fluid infusion rate in millilitres per hour. Finally, for every $10 \mathrm{~kg}$ above $80 \mathrm{~kg}$, an additional $100 \mathrm{ml} /$ hour is required ${ }^{120}$. This simple method of calculating the initial fluid infusion rate in adults $(>40 \mathrm{~kg})$ enables clinicians to focus on applying basic critical care principles to titrate the fluid infusion rate based on a combination of various end points (for example, lactate levels, extent of case deficit, mixed venous oxygen saturation, central venous pressure and mean arterial pressure) 
that are centred on a target urine output. A recent in silico validation study of 100,000 simulated patient weights and burn sizes determined that the Rule of Tens provides a reasonable initial fluid infusion rate across the entire spectrum of burn sizes and patient weights $>40 \mathrm{~kg}^{120}$. However, traditional methods of deriving the initial fluid infusion rate should probably be used in those patients at the extremes of weight. For those $<40 \mathrm{~kg}$ in weight, especially paediatric patients, the initial rates will be higher than for those calculated based on $4 \mathrm{ml} / \mathrm{kg}$ per \%TBSA burned. For those $>130 \mathrm{~kg}$ in weight, the initial rates will be lower than those calculated based in $2 \mathrm{ml} / \mathrm{kg}$ per \% TBSA burned.

\section{Screening}

The concept of screening in a patient with a burn injury is multifaceted given the complexity of the different physical and psychological elements involved and our growing understanding of functional recovery after injury. For example, screening for multidrug-resistant microorganisms early after admission can inform antimicrobial choice in the event of an infection. Standardized predictive formulae can provide estimates of the risk of developing complications such as acute kidney injury ${ }^{121,122}$ and ARDS $^{123}$ after burn injury, but success with their use has been variable. The optimal smoke inhalation screening tool should be highly sensitive to avoid airway loss or respiratory failure due to missed diagnosis but must also be specific to avoid unnecessary intubation. Indeed, unnecessary intubation is associated with increased rates of ventilator-associated pneumonia and other upper airway complications including vocal cord and tracheal injury ${ }^{124}$. Identifying which patients require early intubation to prevent loss of airway after smoke inhalation represents an ongoing area of equipoise ${ }^{109}$. Unfortunately, biomarker identification for the development of predictive clinical decision support tools has so far eluded us.
Additionally, given what we know about the risks of emotional and psychological ramifications after burn injury $^{125}$ (see below, Quality of life), early screening for depression, acute stress and even substance use disorder may facilitate timely psychological and social interventions that will reduce the likelihood of prolonged mental health issues ${ }^{126}$. Emerging data about increased use of health service resources by patients prior to a burn injury suggests an unmet opportunity for injury prevention ${ }^{127}$; furthermore, knowing that a patient has frequently sought emergency care prior to a burn injury could indicate risk of mental health sequelae that warrants targeted intervention.

\section{Management}

Over $95 \%$ of fire-related burn deaths occur in low- and middle-income countries ${ }^{128}$, where centres with burn expertise are sparse (BOX 3). Even in the USA, access to specialized burn care has been repeatedly found to be limited, with up to $20 \%$ of the US population living $>2$ hours by ground or air from a verified burn centre ${ }^{105}$. As such, training of health-care workers at basic-level facilities is essential to reduce death and disability from inadequately treated burns, especially under austere and low-resource conditions, including in low- and middle-income countries, war zones and mass-casualty incidents ${ }^{129,130}$.

For all minor burn injuries, the steps of first aid (BOX 4) are sufficient treatment; these steps are also recommended for the immediate treatment of more severe burn injuries. Indeed, in patients with more severe burns, phase I of the acute management includes the aforementioned primary and secondary surveys ${ }^{131}$. After the acute management phase, four major components of care follow upon admission to a burn centre: resuscitation, burn wound coverage, critical care and/or supportive care, and rehabilitation ${ }^{132}$ (FIG. 7). Another important
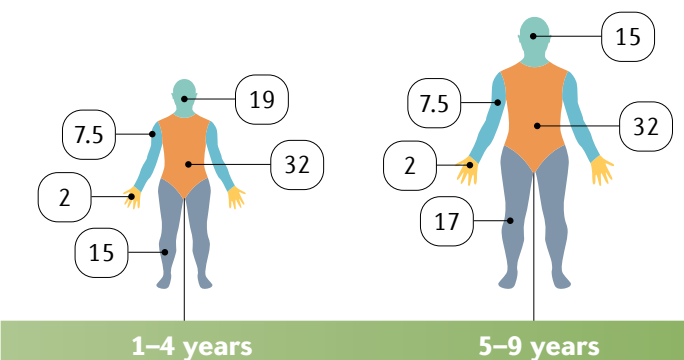

5-9 years

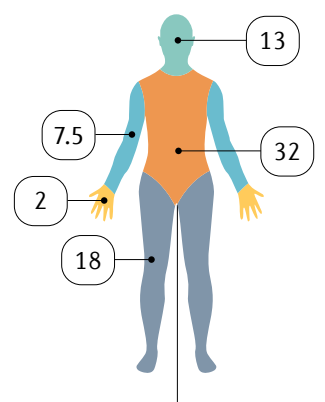

10-14 years

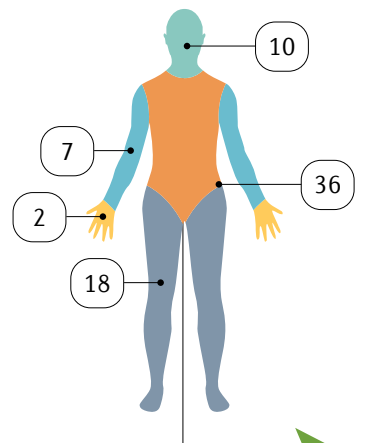

$>16$ years

Fig. 6 | Lund and Browder diagrams for estimating burn size in terms of TBSA. In adults, the 'Rule of Nines' (that is, using multiples of 9) is used to assess the proportion of the total body surface area (TBSA) affected and to help guide immediate treatment decisions, such as amount of fluid resuscitation, that are based on the size of the burn injury. However, owing to different head to body size ratios, the proportion of the TBSA affected in children is estimated differently; the Rule of Nines is inaccurate. Another challenge is the body habitus. For example, the Rule of Nines and the estimate that each hand comprises $1 \%$ of the TBSA are inaccurate in patients who have obesity or cachexia ${ }^{265}$. The body areas are separated by colour and the numbers are percentages of the TBSA and include front and back coverage; for example, '32' in the diagram of the trunk relates to the chest, abdomen and back that make up 32\% of the TBSA. The hand, including the palm, fingers and back of the hand, represents $2 \%$ of the TBSA and can be a useful tool for quick calculation of the size of a burn - especially irregularly shaped scald burns ${ }^{266}$. 


\section{Box 3 | Burn care in low-resource areas}

Advances in our understanding of burn pathophysiology and management have resulted in improved burn care. Unfortunately, the majority of burn injuries occur in resource-challenged or austere environments (RAEs), where even basic burn care may not be available. Furthermore, preparation for potential mass casualty events must include plans for resource limitations, even in normally plentiful regions. The importance of this topic led the American Burn Association to sponsor a series of publications providing guidelines on burn care in RAEs ${ }^{251,252}$.

Some key factors to consider in RAEs specific to burn care are: the knowledge level of the care providers; access to resuscitation fluid; and access to wound care. Ensuring that the 'burn team' is aware of the goals of treatment and the current standards in burn care is essential in any setting to ensure optimal outcomes. Indeed, participants in a burn disaster training course scored only $30 \%$ before training, a score that increased to $65 \%$ after training ${ }^{253}$. Work on education in RAEs has been undertaken by a number of organizations, including the Canadian Network for International Surgery (CNIS) and Interburns. The CNIS developed and published a standardized course for burn care (Essential Burn Management) that was developed and piloted in Ethiopia and Tanzania to meet local provider needs, and has increased knowledge in participants ${ }^{254,255}$. Interburns has published Essential Burn Care, a resource for burn care education in $\mathrm{RAEs}^{256}$. More work needs to be undertaken to ensure that these materials are evaluated and appropriately disseminated.

Another unique aspect of burn care is the need for ongoing resuscitation in patients with major burn injuries. Often RAEs do not have access to intravenous fluids and, accordingly, interest in using oral rehydration solutions has increased ${ }^{257-259}$, most notably the WHO solution, which comprises anhydrous glucose and salts in a prepared package to which water can be added. Current guidelines suggest that oral rehydration solutions can be used for burns up to $40 \%$ TBSA in size, and as an adjunct for larger burns; although a porcine study has shown improved renal function ${ }^{259}$ with such solutions, optimization in humans is still needed. A final factor specific to burn care is the need for antimicrobial dressings. The complex, silver-based, moisture control dressings used in high-resource environments may not be available; locally available alternatives such as Dakin's solution or iodine-based solutions should be considered $^{260,261}$. contributors to predicted mortality. The Modified Baux score includes inhalation injury (present or absent), and is now the most widely accepted outcome predictor to date; it is applicable to patients of a wide range of age, including children ${ }^{133}$.

Burn outcomes should be validated against benchmarks and this concept was introduced by a multicentre trial (Glue Grant study) ${ }^{134}$. More importantly, the ABA is actively working on a prospective database that will assess outcomes and compare the outcomes of a single centre against those of other burn centres within the ABA burn centre community, enabling objective evaluation of outcomes following burn injury.

\section{Special considerations}

Paediatric patients. In general, the care path of a paediatric patient with a burn injury is similar to that of an adult. The initial approach is based on the principles of primary and secondary survey. However, the predominant differences in the care of a paediatric patient with a burn injury stem from the fact that, in children, the head represents a larger proportion of the TBSA and from the reduced overall physiological reserve of children. Newborn babies, for example, have a bigger head to body ratio with correspondingly smaller legs. Additionally, because children have limited glycogen stores, they should be given weight-based maintenance intravenous fluid in the form of $5 \%$ dextrose in $4.5 \%$ normal saline in addition to burn resuscitation fluid. The most important aspects of the care of a paediatric patient, as in adults, are to close the wound, treat hypermetabolism and plan for the long-term outcome.

aspect of planning burn care is predictors of outcome. One of the unique characteristics of burn injury is its reliable dose-response between the extent of the burn and outcome (that is, the larger the burn size, the worse the outcome). The Baux score combines the effect of the extent of the burn injury with patient age, and was described half a century ago. The Baux score is used to predict mortality following burn injury, with patient age and burn size (percentage TBSA) being equal
Elderly patients. In patients $>65$ years of age, who have the worst outcomes after burn injury, pre-existing frailty is a major determinant of outcome ${ }^{135}$. Elderly patients have a unique acute phase response after burn injury that is characterized by decreased organ perfusion and oxygenation ${ }^{136}$. Furthermore, elderly patients are particularly prone to infection, mental health alteration and malnutrition, all of which are associated with increased morbidity and mortality ${ }^{137}$.

\section{Box 4 | First aid}

The immediate care for a patient with a burn injury depends on the cause of the injury (thermal, chemical or electric), the location of the injury and the availability of care resources. If the burn occurs at a remote place, first aid will likely be conducted by a bystander or person without medical training. The first step is to stop the exposure and move the patient into a safe area, which might include getting the person out from a trapped situation (for example, in a vehicle). If the individual's clothing is on fire, suffocation to extinguish the fire is recommended ${ }^{262}$ (by rolling and covering the burning clothing); otherwise, water or another fire extinguisher should be used. Next, the injury site should be flushed with cool water. This not only extinguishes the fire but also cools the wound and reduces the convection of heat and pain. However, use of cool water in this way increases the risk for hypothermia; accordingly, patients should be wrapped in clean dry blankets. Clothing and jewellery should be removed. For a chemical burn, copious flushing with water is recommended, but increases the risk of contamination of rescuers. Use of neutralizing agents is contraindicated as they induce heat. For electrical injures, stopping the current or using an insulator is important prior to touching the patient. Once these steps have been achieved, rescue teams or emergency services can be alerted and usual primary and secondary surveys can commence (see Diagnosis, screening and prevention). Wound care at the site of the accident involves removing burnt textiles, which can be replaced by clean sheets to reduce the risk of contamination of the wound and to maintain body temperature. Use of 'home remedies' such as butter, lemon, toothpaste, hydrogen peroxide ointments or onions is not recommended as many will further damage tissue. Transport principles to the local hospital vary according to the local geographic situation, but air transport may be recommended when the road transport time exceeds 2 hours or $100 \mathrm{~km}$. 


\section{Phase I}

Initial assessment and triage

- Stop the burning process

- Remove patient and providers from harm

- Primary survey (airway, breathing and circulation)

- Secondary survey (assess other injuries, estimate percentage TBSA affected)

- Begin resuscitation (calculate initial fluid rate using either $2-4 \mathrm{ml} / \mathrm{kg}$ per 24 hours

to estimate 24-hour volume or calculate initial fluid rate using Rule of Tens)

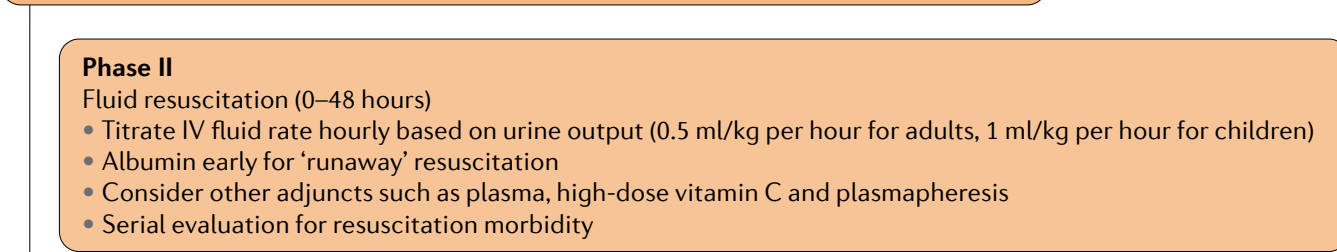

\section{Phase III}

Burn wound coverage

- Use of topical antimicrobial creams or dressings to prevent infection

- Surgical debridement, burn wound excision and autografting or temporary coverage with skin substitute

- Optimize conditions for wound healing (haemodynamics, organ support and nutritional support)

- Treat hospital complications and provide organ support

- Nutritional support

Burn injury

\section{Phase V}

Rehabilitation

- Proper limb positioning to prevent contractures

- Aggressive rehabilitation - active range of motion and exercise

- Anabolic agents (oxandrolone) and catabolism-reducing agents (propranolol)

- Psychosocial support

- Return to work and work strengthening programmes

Fig. 7 | The phases of burn care. Acute care for severe burns can be compartmentalized into five distinct phases that overlap during the first days to weeks after burn injury. Phase I is the initial assessment and triage, in which the injurious cause is removed and the primary and secondary surveys are conducted. Phase II is focused on fluid resuscitation to address hypovolaemia. In phase III, the wound is covered to promote healing and reduce infection risk. Phase IV focuses on supportive or critical care. If the patient survives, phase $V$ of care focuses on rehabilitation, which includes physical and mental health support to enable the patient in returning to regular life. IV, intravenous; TBSA, total body surface area.

\section{Phase II: resuscitation}

Burn shock, which combines hypovolaemic, distributive and cardiogenic features, occurs in the initial 48 hours secondary to the dysregulated inflammatory response after burn injury, and is characterized by a diffuse capillary leak wherein losses of proteins, electrolytes and plasma further reduce intravascular volume, impair end-organ perfusion and produce cellular dysoxia (aberrant cellular oxygen metabolism). As discussed previously, a number of factors contribute to the magnitude of the response, the combination of which contributes to considerable complexity and requires an individualized approach. In general, those with burn sizes $>20 \%$ TBSA will require fluid resuscitation. However, patients with smaller burns may also require fluid resuscitation under circumstances in which other insults exists, such as electrical injury, smoke inhalation or concomitant trauma. The ultimate goal of fluid resuscitation is to maintain end-organ perfusion while avoiding resuscitation-related morbidity such as extremity, abdominal and orbital compartment syndromes (conditions characterized by acute increases in pressure in specific compartments, requiring emergent decompression to avoid cell death).

The initial fluid of choice is a balanced crystalloid, most commonly warmed Ringer's lactate solution ${ }^{38}$. However, in most patients with severe burns, exclusive use of a crystalloid solution can result in over-resuscitation (that is, a sustained high-volume crystalloid resuscitation that is associated with the development of resuscitationrelated morbidity). To address this situation, 'colloid rescue $^{138}$ using other adjuncts such as albumin or plasma has been suggested. 
Colloids: albumin or plasma. A recent meta-analysis of burn resuscitation outcomes ${ }^{139}$ indicated that albuminaugmented resuscitation with $5 \%$ albumin as early as 8 hours after burn injury in those who are projected to receive a massive resuscitation (defined as those needing $>1,500 \mathrm{ml}$ per hour for 2 hours or $>250 \mathrm{ml} / \mathrm{kg}$ in 24 hours) enables the infusion of a lower total volume of crystalloid in the first 24 hours. A previous randomized controlled trial has shown that the use of plasma, an alternative to albumin, reduces 24 -hour fluid volumes, decreases intra-abdominal pressure and reduces subsequent development of abdominal compartment syndrome compared with administration of crystalloid ${ }^{140}$. Additionally, plasma seems to have important advantages related to preserving the microvascular endothelium by maintaining the cell wall glycocalyx compared with other resuscitative fluids ${ }^{141}$. Unique to the use of plasma are the known risks of pooled blood product transfusions, which include transfusion-related lung injury. However, recent improvements in screening have mitigated this risk ${ }^{142}$.

Vitamin $C$. The antioxidant and reactive oxygen scavenging ability of vitamin $\mathrm{C}^{143}$ prompted interest in its use during burn resuscitation. Initial animal studies were favourable, with decreased fluid requirement, oedema and capillary leak, and reduced lipid peroxidation, compared with crystalloid alone $\mathrm{e}^{143,144}$. In a small randomized trial in humans, high-dose vitamin C reduced 24-hour crystalloid fluid volumes and improved some ventilation and oxygenation parameters without a significant difference in mortality ${ }^{145}$. Recent clinical data on vitamin $\mathrm{C}$ in patients with ARDS also seems positive ${ }^{146}$, suggesting that vitamin $\mathrm{C}$ might have a beneficial effect in patients with burn injury. However, conflicting data on renal safety ${ }^{147,148}$ and falsely elevated point-of-care glucose levels have prevented adoption of resuscitative vitamin $\mathrm{C}^{149}$ and highlight the need for robust clinical trials of its use in patients with burn injury.

Blood purification strategies. Blood purification strategies are appealing because they aim to remove circulating inflammatory mediators ${ }^{150}$. Indeed, retrospective studies of therapeutic plasma exchange (TPE) have demonstrated reduced resuscitative volume needs, improved mean arterial blood pressure, increased urine output and decreased lactate levels ${ }^{150,151}$. Unfortunately, a single TPE treatment requires high volumes of either donor plasma or colloid, and consequently TPE is used by few centres due to cost and the resources required.

A more practical alternative to TPE may be haemofiltration. High-volume haemofiltration (HVHF) (doses $>70 \mathrm{ml} / \mathrm{kg}$ per hour) may benefit patients in shock and can be used as an adjunct in those with burn shock ${ }^{152,153}$. A recent multicentre trial in patients with burn injuries demonstrated that HVHF was effective in reversing shock and improving organ function ${ }^{154}$. Another trial demonstrated that HVHF decreased the incidence of sepsis, duration of vasopressor treatment needed and mortality in patients with severe burn injuries ${ }^{155}$. This study demonstrated a significant decrease in circulating inflammatory markers in the HVHF group compared with controls ${ }^{155}$. These blood purification strategies may not be practical in a resource-constrained environment.

\section{Phase III: burn wound coverage}

In the first half of the 20th century, burn injuries were managed expectantly and, unfortunately, patients frequently succumbed to overwhelming sepsis while waiting for their burn eschar (scab) to slough, or for their open wounds to close by secondary intention (that is, allowing the burn injury to heal on its own). Topical antimicrobials slow the septic process ${ }^{156}$ and have been instrumental in preventing those with larger, more-severe burns from succumbing to sepsis. However, improvements in survival and shortened length of hospital stay were only realized after the introduction in the 1970s of early excision and grafting ${ }^{157-159}$.

Prophylactic systemic antibiotics are not used in the care of patients with an acute burn. However, topical antimicrobials have been the mainstay of nonsurgical burn treatment. Topical agents take a variety of forms: creams, ointments, liquids and impregnated dressings. The majority of dressing protocols use silver in some form owing to little clinical resistance by microorganisms. The literature to support one type of dressing over another is of variable quality and, accordingly, no clear consensus favouring one dressing is available; each regional burn unit tends to favour a particular dressing based on availability, personnel preference and historical experience ${ }^{160}$. Regardless of the dressing chosen, some form of antimicrobial activity is desirable ${ }^{161}$.

Early excision, the gold-standard treatment, attenuates the hypermetabolic state and removes the biological nidus for infection, thereby reducing the risk of burn wound sepsis. Early autografting (that is, where skin is transplanted from a healthy (donor) site on the patient to the burned part) then builds on these improvements by rapidly closing excised wounds, further reducing infection risk, decreasing pain and enabling earlier mobilization. However, the optimal timing for 'early' excision is debated; for example, whether a patient undergoes surgery during the first day after burn, or 2-3 days later after completion of the 24-hour resuscitation, remains unclear. Regardless, the principle of excision and grafting is clear: close the wound. If a patient has good donor skin sites and is stable in resuscitation, autografting should be conducted while the wound bed is fresh and uncontaminated. If there are concerns about the viability or bacterial load on the wound bed and/or the patient's stability, allografting (that is, using skin from another person (usually cadaveric)) should be used to temporarily cover the debrided wound. The surgical approach is to leave no full-thickness burned tissue behind and debride down to viable tissue.

Although many surgeons believe excising and covering the largest areas, such as back and trunk, should be performed first, in some centres the face and hands are covered first before transitioning to the body. We (M.G.J., S.L. and N.S.G.) believe that excision and coverage of the back and trunk have priority followed by the joints, legs and arms. Occasionally, valuable uninjured patches of potential donor sites can be 'saved' for use on important functional and cosmetic areas such 
as the hands and face. Our approach is based on the hypothesis that large burned areas contribute most to and augment hypermetabolism and inflammation and are, therefore, directly linked to poor outcomes. Once the larger areas have been covered, 'healed' skin can be harvested for delicate areas such the face, eyelids and hands; although cosmetic outcomes may not be optimal (owing to the reduced thickness of the skin), later reconstructions can be addressed once the patient is stabilized and out of hospital. Regardless of the order, skin grafts are usually meshed (that is, processed to 'punch' apertures onto the graft, allowing it to expand in size and permit ingress of topical agents and egress of serous fluid or blood) for the back trunk, legs and arms; sheet grafts (that is, donor sites that are excised and used as-is) are used for cosmetic areas such as the hands, face and neck.

Autologous split-thickness skin grafts. The gold-standard burn coverage is autologous split-thickness skin grafts (STSGs). Uninjured skin is harvested using a dermatome instrument, and the STSGs are transferred to an excised burn site. In burns covering $>30-40 \%$ TBSA, available uninjured skin may be insufficient to allow definitive coverage in a single operation. In such cases, STSGs are frequently meshed and expanded to cover a larger excised area (FIG. 8); this approach trades aesthetic and functional outcomes for rapid closure and reduced donor site morbidity. For larger burns, usually $>60 \%$ TBSA, mesh ratios $>1: 3$ may be required, but are very technically challenging to use owing to fragility of the meshed skin, often resulting in substantial portions of the 'grafted' areas remaining open, necessitating overgrafting with allograft to functionally close the wound ${ }^{162}$. A solution to this issue is provided by Meek meshing technology, which expands the skin graft up to a ratio of 1:9 (FIG. 8). The expanded mesh can cover larger areas and the morbidity of poor scarring (see below) can be alleviated by combining the Meek technique with a dermal regenerative matrix and/or spray of a cell suspension ${ }^{163}$, which can assist in closing the wound.

Failure of engraftment is typically the result of inadequate recipient site excision, shear stress or wound infection ${ }^{164}$. Definitive coverage following large burns rapidly exhausts available donor skin and must, therefore, be performed in stages. When the excised burned area exceeds the available donor skin, 'temporary coverage' is needed to both permit donor site re-epithelialization in anticipation of re-harvest and to avoid the complications of open excisions. Common skin substitutes include cadaver skin (allograft) and porcine skin (xenograft), which provide temporary coverage for up to 14 days before inevitable rejection ${ }^{36}$. STSG quality, quantity and the ability to re-harvest are functions of donor site skin thickness, harvested STSG thickness and the interval between re-harvests.
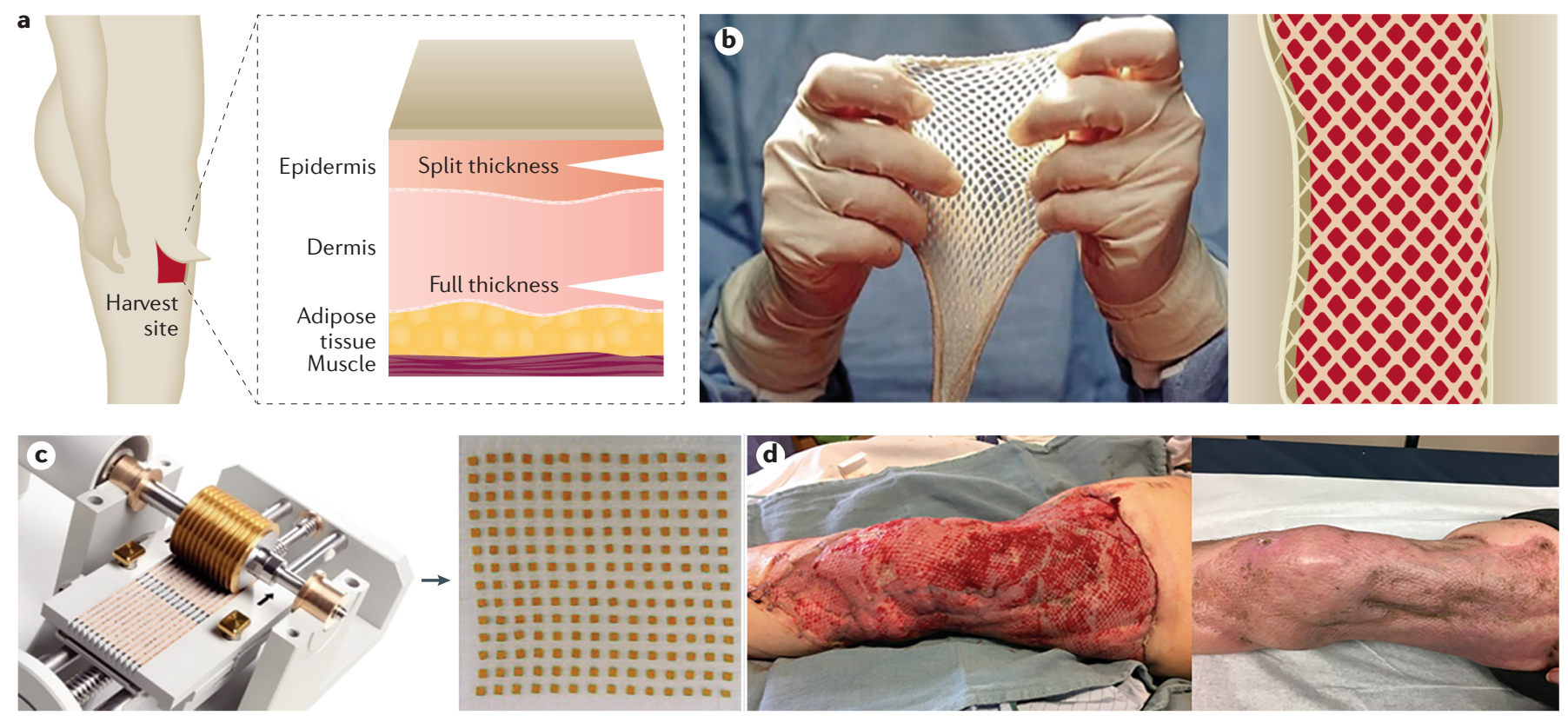

Fig. 8 | Autologous split-thickness skin grafts. For a patient with a severe burn injury to survive, the burn wounds need to be excised and covered. Temporary measures using allograft or various biological substitutes are available but, at this time only the patient's skin (autologous) can permanently accomplish coverage. Several graft methods are available to cover burn wounds using autologous skin. a | Sheet grafts are the most aesthetically pleasing but require a lot of skin to cover wounds and, therefore, are usually reserved for small burns or for skin grafts to complex and important areas such as the face, hands and breasts. Full-thickness sheet grafts are reserved for smaller defects (usually lower eyelids and re-occurring upper eyelids) and play a more important part during the reconstructive phase. These are harvested using a dermatome.
A split-thickness graft can be placed as a sheet graft or used for meshing. b| Use of meshed split thickness skin grafts is usually the method of choice to cover larger areas. The goal of meshing skin is to expand the donor skin to obtain greater coverage; skin can be meshed in ratios of 1:1.5, 1:2, 1:3, $1: 4$ or 1:6. Although increased meshing increases the coverage size, meshed skin becomes increasingly fragile. $\mathbf{c} \mid$ An alternative technique is the Meek technique, in which skin squares can be spread out to a large extent and added to the wound bed, covering large areas (up to a ratio of 1:9). This technology is reserved for extensive burns for which donor sites are sparse. $\mathbf{d} \mid$ A freshly meshed split-thickness skin graft (left) and its healing over time (right). Images in part c courtesy of R. Nijlant, Humeca B.V., Netherlands. 

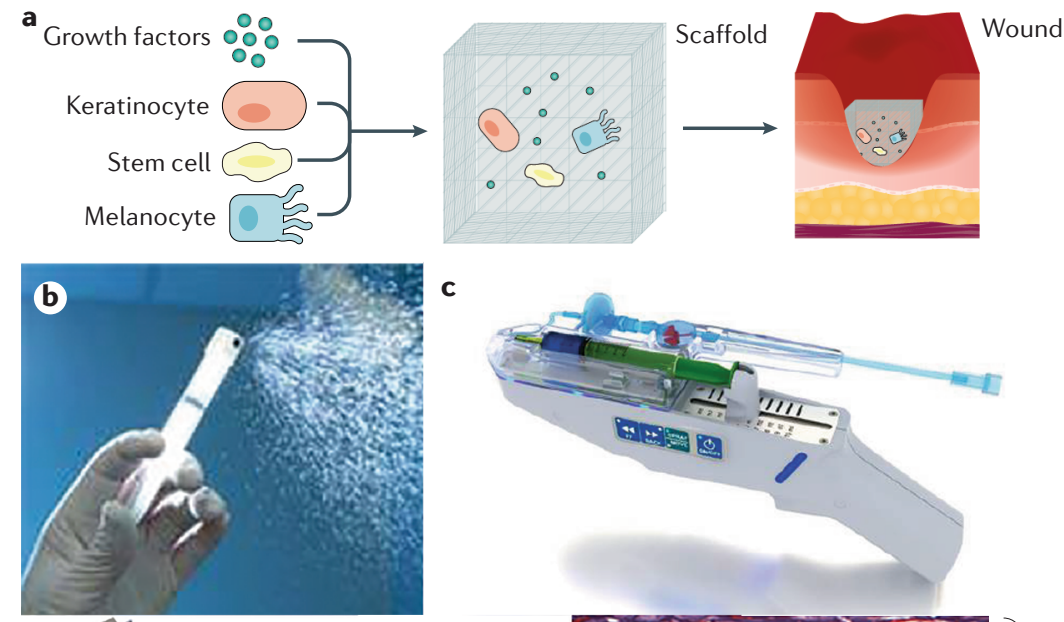

c
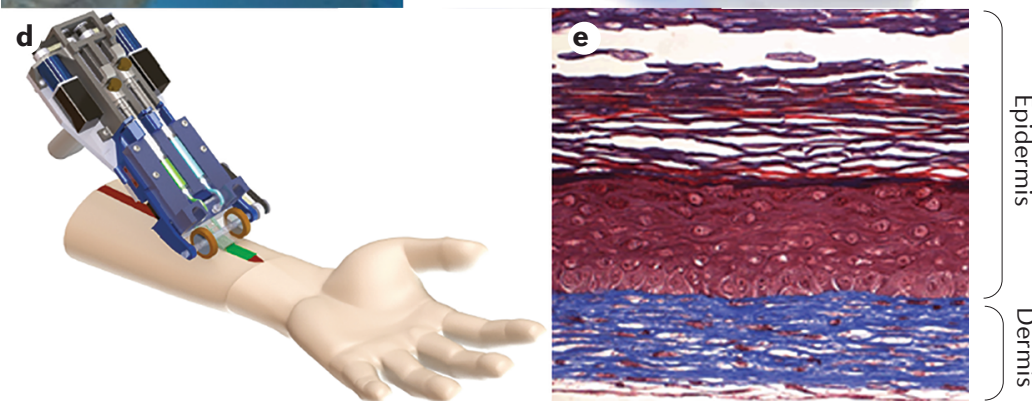

Fig. 9 | Skin substitutes. Skin substitutes have undergone development over the past decade from temporary materials used to induce wound healing towards permanent tissue-engineered materials that offer definitive healing. This figures reflects a summary of some promising skin substitutes or treatments to induce wound regeneration. a| The common principle of skin substitutes is to the deliver proteins, growth factors and/or cells via a delivery vehicle or matrix that will then be integrated into the wound and form new autologous skin. $\mathbf{b} \mid$ ReCell is not a composite skin substitute, but a device that sprays a cell suspension of skin cells including epithelial cells, fibroblasts melanocytes and other resident cells onto the wound or grafted area to improve healing and scarring. c| The skin gun (RenovaCare) delivers autologous cells and/or stem cells to wounds to improve wound healing. Although this is a promising approach, no clinical trials or substantial evidence have been reported that indicate that the skin gun will enter the clinical arena. $\mathbf{d} \mid$ Recently, a hand-held device has been designed that can print 3D autologous skin ${ }^{248}$. This device is based on microfluidic technology and can deliver cells specifically and accurately in an 'ink', which serves as a matrix. e|A skin substitute using autologous cells from a healthy donor and a matrix shows self-assembly of dermal and epidermal structures. Although the ideal tissue-engineered skin derivative has not been described, this technology will change the way burn care is delivered and will improve acute and long-term outcomes. Image in part b courtesy of Avita Medical. Image in part c courtesy of RenovaCare Inc. Part d provided by A. Guenther (University of Toronto, Canada), and adapted with permission from REF. ${ }^{248}$, Royal Society of Chemistry. Panel e is reprinted from REF. ${ }^{267}$, CC BY 4.0 (https://creativecommons.org/licenses/by/4.0/).

Artificial skin substitutes. In cases in which autologous skin is insufficient, thin and/or frail, artificial skin substitutes ${ }^{36}$ can be used (FIG. 9). Composed of various biological, synthetic and biosynthetic materials, artificial skin substitutes can provide either temporary or permanent coverage of open wound $s^{165,166}$. In manufacturing skin substitutes, the scaffold is always taken into consideration. Scaffolds are 3D structures that act as a substitutes for extracellular matrix, providing the framework for neovascularization, cell adhesion, proliferation and differentiation. In addition, growth factors and cells can be added to the scaffold to enhance healing ${ }^{167,168}$. Many variations are available, although all skin substitutes should demonstrate the following: resistance to shearing forces when handled; structure and function of normal skin that protect from fluid loss and infection; ability to act as a scaffold and enable cells to proliferate for wound healing; non-inflammatory, nontoxic and non-immunogenic composition; and affordability ${ }^{169,170}$. For example, Skin TE (Polarity) is a technology where a skin biopsy is digested, sent to the manufacturer and then incorporated into a gel that contains epidermal and dermal elements, as well as skin appendages, that is directly applied to the burn wound ${ }^{171}$. Additionally, there are different classification systems for skin substitutes $^{172}$. The Davison-Kotler classification ${ }^{173}$ categorizes skin substitutes based their composition (cellular ${ }^{174}$ or acellular ${ }^{172}$ ), whether they are single layer or bilayer, their structure (epidermal (derived from human placenta $^{175,176}$, dermal or composite (for example, a composite skin substitute composed of a bovine collagen, glycosaminoglycan bilayer and with a silicone layer that acts as the epidermis $\left.{ }^{172}\right)$ ), the type of biomaterial used (synthetic, biosynthetic or biological), and the duration of cover (permanent, semi-permanent or temporary) ${ }^{177}$.

Skin substitutes have also been used as biological or bioactive dressings that facilitate re-epithelialization of superficial partial-thickness burns underneath the skin substitute, ultimately functioning as definitive coverage $^{178}$. In selected patients with large burns, cultured epithelial autograft (CEA) can be used (TABLE 1). Although CEA can be used to cover large burns, it is limited by fragility, cost and the technical expertise required for optimal engraftment. When selected, use with an acellular dermal matrix may improve CEA outcomes ${ }^{179}$. Additionally, Epicel is cultured skin derived from an autologous skin biopsy specimen; autologous keratinocytes are incubated for 2-3 weeks and grown into sheets of epidermal autograft.

Scarring. A central part of the long-term outcome after burn injury is scarring; scars are optimally flat and minimally discoloured. After burn injury, normal scars are characterized by increased collagenase activity, lower TGF $\beta$ expression and macrophages with a predominantly $\mathrm{M} 1$ phenotype, which promotes $\mathrm{T}$ helper 1 cell subsets. However, burns can result in the development of a pathological scar. In particular, deep partial-thickness or full-thickness burns take longer to heal, which results in an increased risk of pathological scarring, especially when combined with a prolonged acute inflammatory phase. Pathological scars are characterized by excess collagen deposition, resulting in a thick, non-pliable defect that can cause itch, pain and contractures, limiting functionality ${ }^{180}$.

Two primary subtypes of pathological scars occur after thermal injury - hypertrophic scars and keloids. Hypertrophic scars are more common in burn injuries and occur in $30-90 \%$ of patients with burn injuries ${ }^{180}$. Delay ( $>3$ weeks) in wound healing increases the risk of hypertrophic scarring ${ }^{180}$, which is typically restricted to the confines of the initial injury and does not recur after excision. Decreased collagenase activity in hypertrophic scars leads to perturbed collagen production and degradation, resulting in bundles of crosslinked collagen oriented 
Table 1 | Example commercially available skin substitutes

$\begin{array}{llll}\text { Name (manufacturer) } & \text { Composition } & \text { Advantages } & \text { Disadvantages } \\ \text { Epidermal: CEA } & & \text { Refs } \\ \begin{array}{l}\text { Epicel (Vericel } \\ \text { Corporation, }\end{array} & \begin{array}{l}\text { CEA from human keratinocytes } \\ \text { embedded in fibrin mesh }\end{array} & \text { Autologous coverage of epidermis } & \begin{array}{l}\text { High cost; limited reliability; } \\ \text { fragile; increased susceptibility to } \\ \text { infections; complex postoperative } \\ \text { care; tendency for blistering; } \\ \text { poor healing }\end{array}\end{array}$

Epidermal: autologous skin suspension or cell spray

ReCell (Avita Medical, Autologous skin suspension Woburn, Massachusetts, produced using minimal donor skin USA) and applied as a cell spray.

\section{MySkin (Regenerys, \\ Cambridge, UK) \\ SkinGun (RenovaCare, Epidermal cells and stem cells Inc., NY, USA) \\ Keraheal (MCTT, Seoul, \\ Korea) \\ Suspension formed of cultured epithelial cells plus fibrin glue \\ Dermal \\ OASIS Wound Matrix \\ (Healthpoint Ltd, \\ Ft Worth, TX, USA) \\ Derived from the submucosal layers of the porcine intestine; contains glycosaminoglycans and growth factors}

\section{Matriderm (MedSkin}

Solutions Dr. Suwelack

AG, Billerbeck, Germany)

NovoSorb BTM

(Biodegradable

Temporizing Matrix)

(PolyNovo, Melbourne,

Australia)

MatriStem (ACell, Inc.,

Columbia, MD, USA)

Integra (Integra

LifeSciences, Plainsboro,

NJ, USA)

Alloderm (Allergan,

Dublin, Ireland)

SUPRATHEL (Polymedics Innovations $\mathrm{GmbH}$,

Denkendorf, Germany)

Made of type I collagen fibre coated with $3 \%$ a-elastin

hydrosylate matrix

Biodegradable polyurethane foam plus a temporary nonbiodegradable polyurethane seal; fully synthetic

Composed of extracellular matrix derived from porcine urothelium

Bovine collagen matrix with a silicone layer

Human cadaveric acellular matrix

Synthetic copolymer $>70 \%$ DL-lactide polymerized with $\varepsilon$-caprolactone and methylenecarbonate

(and
Induces acceleration of reepithelialization and wound healing for wide meshes, most likely to improve appearance of scar

Promotes re-epithelialization

Expansion ratio of skin donor site to treatment surface area of $\sim 1: 20$

Facilitates epithelial cell attachment

No RCT data

Dermal element to improve scar appearance

Porcine composition; high cost
Can be used as single-stage procedure with split-thickness skin grafts; very good outcomes in initial clinical trials

Low cost; improves appearance; good No RCT data results in initial clinical trials to infections
High cost; no dermal substitute;

limited to more superficial burns

No RCT data

\section{No RCT data}

Possible increased susceptibility
Provides barrier protection

Most-studied dermal replacement matrix; improves scarring and skin appearance; used in acute and reconstructive surgery; efficacy shown in RCT

Improves scarring

Promising results in initial clinical studies

\section{No RCT data}

High cost; possible increased susceptibility to infections

\section{Composite}

Self-Assembled Skin

Substitute (SASS)

(Loex, Quebec, Canada) Epifix (MiMedx, Marietta,
GA, USA)

Cultured skin substitute (prepared at Shriners Hospitals for Children, Cincinnati, OH, USA)
Reconstruction of a fully autologous bilayered skin substitute

\section{Composed of dehydrated} amniotic and chorionic membrane containing collagen, connective tissue, cytokines and growth factors

Autologous keratinocytes and fibroblasts from patient biopsy, combined into a bilayer with bovine collagen matrix
No exogenous scaffold or biomaterial needed; good scar development; autologous

Improves wound healing and regeneration

Good scaring; epithelial and dermal elements
Requires a 31-day production period; very high cost; limited clinical data

Not a skin substitute per se; no RCT data

High cost; long production time; does not include melanocytes

CEA, cultured epithelial autograft; RCT, Randomized controlled trial. 
parallel to the epidermal surface ${ }^{180}$. In particular, mature type I collagen expression is reduced and type III collagen is over-synthesized ${ }^{181}$. Additionally, hypertrophic scars have higher TGF $\beta$ and PDGF expression, macrophages with a predominantly M2 phenotype, which promotes T helper 2 cell subsets, and increased myofibroblasts compared with normal scars. Hypertrophic scar colour ranges from hypopigmented to hyperpigmented.

By contrast, keloid scars are occur mainly in those with darker skin pigmentation ${ }^{180}$ and develop several months or years after the initial insult. Keloid scars are raised fibroproliferative lesions composed of disorganized bundles of type I and type III collagen. They exhibit tumour-like properties and are characterized by a dependence on anaerobic glycolysis to produce metabolites necessary for cell proliferation ${ }^{182}$. Furthermore, keloid scars demonstrate uncontrolled growth, invasion of normal tissues and recurrence despite treatment ${ }^{183}$. Importantly, persistence of the inflammatory and proliferative phases of wound healing are likely to predispose patients to keloid scarring and results in a scar that grows beyond the initial burn wound ${ }^{180}$. Keloid scar pigmentation can be increased or decreased compared with normal skin but is typically increased compared with the normal 'baseline' skin pigmentation of the patient.

Both keloid and hypertrophic scars detrimentally affect quality of life and are managed with a combination of intralesional pharmacological therapy, contracture release, scar excision, scar massage and laser therapy depending on the scar presentation.

\section{Phase IV: supportive care}

Given that wound closure is tied to improvements in both mortality and morbidity ${ }^{184}$, supportive critical care that promotes optimal conditions for wound healing should be the clear focus of post-engraftment care. All aspects of care should be focused on optimizing the conditions for wound healing while preventing common hospital-acquired complications (for example, venous thromboembolic complications, stress ulcers, hospitalacquired and ventilator-associated pneumonia, catheterrelated bloodstream infection and catheter-related urinary tract infection). Optimizing the conditions of wound healing includes haemodynamic support in the early phases (fluid resuscitation and occasional vasopressor support), optimization of nutritional support and use of pharmacological adjuncts to mitigate the impacts of hypermetabolism while promoting healing (such as propranolol and oxandralone) ${ }^{36}$. At every phase of care, adequate pain control with the appropriate use of opioids and opioid-sparing adjuncts are paramount.

\section{Phase V: rehabilitation}

Despite an appropriate focus on medical and surgical interventions, planning for reconstruction and rehabilitation after major burn injury should begin at admission. To ensure a focus on 'functional survival', multidisciplinary burn teams are involved early upon hospitalization. Within these teams, physical and occupational therapists integrate rehabilitative principles throughout the continuum of burn care. Simple interventions such as proper limb positioning, splinting and incremental weight-bearing even in mechanically ventilated patients, can reduce burn contracture, minimize oedema and improve functional outcomes ${ }^{185,186}$. A multicentre observational study in 307 patients with acute burn injuries demonstrated a significant link between increased duration of therapy and a decrease in the development of burn wound contractures ${ }^{187}$ - the first study to demonstrate such a link between rehabilitative interventions and patient outcomes.

Occupational therapy, physical therapy and mobilization are crucial elements of care after burn injuries. These therapeutic approaches can improve joint stiffness, prevent heterotopic ossification (the formation of bone within the tissue where bone should not form) and improve muscle and tendon function. Additionally, the hypermetabolic response can be reduced with exercise ${ }^{7}$. Accordingly, exercise and therapy should be initiated as early as possible and mobilization should be started immediately after admission, keeping movement restrictions after surgery as brief as possible ${ }^{49}$. Additionally, positioning of patients with burn injuries is imperative and anti-contracture positioning is central in the prevention of contraction and in improving graft take.

Not surprisingly, the psychosocial cost of severe injury is high on patients and their families ${ }^{188}$. For this reason, it is advisable to fully integrate social workers, vocational counsellors and psychologists into the multidisciplinary burn team. Phase V has been neglected or even ignored for some time; it was only recently that we learned about the essential importance and value of good quality long-term outcomes after burn injury ${ }^{125,126}$.

\section{Quality of life}

Health-related quality of life (HRQOL) is a multidimensional concept that reflects an individual's perception of how a condition affects health-related domains for physical, psychological, emotional and social wellbeing that considers quality of life in relation to other health-related components (personal and environmental factors) ${ }^{189}$. HRQOL is an increasingly studied outcome after burn injuries, in both the short and long term ${ }^{190}$. Indeed, several tools can assess HRQOL in adult patients after burn injuries. For example, the Burn-Specific Health ScaleBrief (BSHS), Medical Outcomes Survey Short Form-36 (SF-36) and EuroQol (EQ-5D) questionnaires are often used $^{191-198}$. The use of both burn-specific and generic instruments provides insight into consequences of burn injuries in themselves and also in reference to other conditions ${ }^{195,198}$. However, emerging data cast doubt on the psychometric quality of several burn-specific instruments ${ }^{199}$. For example, the SF-36 domains were shown to be more sensitive to change than BSHS-B domains 1 month after burn injury in one study ${ }^{200}$. In addition, the specific psychometric properties of the BSHS-B are not yet available, including test-retest reliability (whereby repeated measurements over time show similar results in unchanged patients), validity of hypothesis testing and item-total correlations (whereby all items of a domain measure the same construct) ${ }^{195}$. Thus, the optimal burn-specific instrument is not yet available. Several new instruments, including the Brisbane Burn Scar Impact Profile ${ }^{196,201}$, the CARe burn scale ${ }^{202}$ and the 
LIBRE survey ${ }^{203}$, warrant further validation. Of special note, instruments developed for surveying patients in high-income countries may not pertain to burn survivors in lower income countries ${ }^{204}$ or those living in rural or remote areas.

Models that can predict recovery of quality of life after burn injury can inform patients and health-care professionals on the expected outcomes, identify patients with expected suboptimal outcomes and enable the tailoring of treatment to optimize outcomes. Also, such models can be used for benchmark purposes ${ }^{205}$. Indeed, prognostication models for HRQOL after burn injury have been developed for use in children and adolescents ${ }^{206,207}$. Spronk et al. ${ }^{208}$ have recently developed a prognostication model for use in adult patients (submitted for publication), which should be further validated in different burn populations.

\section{Adults}

Two studies have indicated that women report worse HRQOL 1 year $^{209}$ and 10 years after a burn injury ${ }^{210}$, but other reports suggest less consistent correlations between female $\operatorname{sex}^{211}$, pain and post-burn substance use disorder and poor HRQOL ${ }^{212}$. Furthermore, although HRQOL improves over time overall, some domains, including physical and emotional role participation, anxiety, depression and pain or discomfort, demonstrate delayed return to baseline after a burn injury ${ }^{212}$. Compared with the general population, patients who sustain severe burn injuries report lower quality of life, especially related to anxiety and depression, and pain and discomfort ${ }^{213,214}$.

With increasing focus on post-intensive care syndrome, an increasingly understood condition in which critically ill patients are recognized to have longstanding physical and cognitive condition $\mathrm{s}^{215}$, health systems must consider the long-term effects of hospital-acquired complications on functional recovery. Patients with burn injuries who experience a urinary tract infection, venothromboembolism, pulmonary complication or acute kidney injury during hospitalization reported lower physical component summary scores on the SF-12 for up to 24 months after the injury ${ }^{216}$. Although in most quality of life studies, follow-up has been limited to 24 months after burn injury, emerging data suggest that patients struggle with functional recovery and HRQOL for as long as 10 years after the injury is sustained ${ }^{210}$. As we become more familiar with the issues that drive long-term functional recovery, use of additional instruments such as Satisfaction with Life (SWL ${ }^{125,217}$, which measures global cognitive judgments of satisfaction with life), Satisfaction with Appearance (SWAP ${ }^{218,219}$, which measures body image dissatisfaction in persons with visible disfigurement), and the Community Integration Questionnaire (CIQ ${ }^{220}$, which measures levels of community integration) provide alternative options for assessing patient perceptions of their HRQOL.

Even patients with minor burn injuries report more anxiety and depression than the general population, despite having overall comparable $\mathrm{HRQOL}^{213}$. A review of 19 studies $^{212}$ showed that burn severity, post-burn depression, post-traumatic stress symptoms, avoidance coping, less emotional or social support, higher levels of neuroticism and unemployment predict poorer HRQOL, suggesting that the mental health aspects in survivors of burn injury are substantial.

\section{Children}

In children after burn injury, the Burns Outcome Questionnaire for children $0-5$ years of age $\left(\mathrm{BOQ}_{0-5}\right)^{203}$ and the $\mathrm{BOQ}_{6-18}\left(\mathrm{REF}_{.}^{221}\right)$ are valuable resources for quality of life assessment. The $\mathrm{BOQ}_{0-5}$ consists of ten scales, ranging from play to appearance, whereas the $\mathrm{BOQ}_{6-18}$ consists of 12 scales addressing physical functioning, emotional health and social functioning including school re-entry. However, the domains in these questionnaires correlate poorly with those of the WHO International Classification of Functioning, Disability and Health ${ }^{222}$, suggesting that further development and validation of the BOQ surveys is required ${ }^{223}$. Indeed, few studies have addressed HRQOL in paediatric patients after burn injuries, especially in young children up to 4 years of age. Burn size and depth, length of hospital stay, presence of facial and hand burns, comorbidity and long recovery time predict suboptimal HRQOL following burn injury in children ${ }^{224}$. Studies in paediatric patients have shown improvements in HRQOL over the first 6 months after injury ${ }^{223,224}$. However, some domains, including 'concern/ worry' and 'appearance' in young children and 'appearance' and 'parental concern' in children 5-18 years of age, show persistent lack of improvement ${ }^{224,225}$. Thus, children and adolescents seem to have continuing problems with changes in appearance, and parents have concerns related to their children after burn injury.

Assessing quality of life in children, especially those $<12$ years of age can be challenging. However, using parents as surrogates may in fact not reliably predict individual perception of quality of life. For example, using the validated questionnaire designed for adolescents (BOQ), adolescents and parents' assessments of appearance, itch and school re-entry differed ${ }^{226}$. Thus, results on parent-reported quality of life should always be interpreted with caution and should ideally be checked with the child.

\section{Employment and school after injury}

Return to work and school are acknowledged to be important outcomes after burn care. Beneficial for individual health, social and societal aspects for individuals, work is not only a source of income but represents evidence of community integration and participation. Two systematic reviews showed that $66-74 \%$ of burn survivors returned to any form of work 1-24 months after the burn injury ${ }^{227,228}$. Several factors predicted return to work, including burn severity, length of hospital stay, age, psychosocial and work-related factors ${ }^{227,228}$. For example, one single-site study in Europe showed a high return to work rate of $92 \%$ in their population, in whom the mean burn size was $8 \% \mathrm{TBSA}^{229}$; systematic reviews of studies in patients with burn sizes in the range 18-22\% TBSA showed lower return to work rates. Regardless, the economic burden of burn injuries is substantial; in one European study the mean number of days absent from work was 59.3 per patient and the mean cost due to loss in productivity was $€ 11,916$ per patient $\left(\mathrm{REF}^{229}\right)$. 
In children, there is minimal literature available on time to school re-entry. One review showed a short time to school re-entry (mean 10.5 days) in children 6-18 years of age after admission to a burn centre for burn injury with a median size of $14.3 \% \mathrm{TBSA}$; longer time to school re-entry was associated with male sex, older age and a longer hospital stay ${ }^{230}$. A recent literature review on school reintegration programmes after burn injury in children showed a wide range for time to school re-entry, and emphasized the critical role of a formal school reintegration programme to facilitate the transition and educating school personnel ${ }^{231}$. Such a programme involves three stakeholders: the injured child, the child's parent(s) or caregiver(s), and the teacher. Indeed, teachers play a central part in keeping up academic skills and facilitating social reintegration after burn injury ${ }^{231}$. Clearly, gaps in knowledge related to our understanding of the barriers to returning to work and school must be addressed.

No data have been published on return to work or school after burn injuries in low- and middle-income countries, although data are emerging. One important issue related to employment is our definition of 'work': in many low-resource communities, individuals have a subsistence form of living in which work may not actually involve a paid job.

\section{Secondary conditions}

Recent publications have addressed long-term medical consequences of burn injury ${ }^{232}$. In a cohort of patients with burn injury in Western Australia, linked hospital and death data indicated that both minor and severe burn injuries result in increased long-term morbidity and mortality in all age groups, as well as increased risk of hospital re-admission, long-term hospitalization and prolonged hospital stay for musculoskeletal diseases ${ }^{233-235}$. Adult patients with burn injuries and patients with trauma showed elevated rates of post-injury admissions for cardiovascular events and an increased incidence of diabetes mellitus-related admissions during the first 5 years after injury ${ }^{236}$. Children with burn injury had a 1.6-times greater rate of long-term mortality than children with no injury ${ }^{237}$.

\section{Outlook}

What will the future of burn care bring? We believe that burn care must change to adjust to the challenges ahead.

\section{Epidemiology and prevention}

In the area of epidemiology, it would be important to know what the exact burden and effects of burn injuries are globally. To better identify needs, treatment, prevention and knowledge translation, capturing data more accurately is imperative, which should then lead to the development of strategies that translate this knowledge into actions. We believe that global agencies such as the WHO and the International Society for Burn Injuries should be integral to initiatives. Indeed, the WHO is piloting an important check-list initiative that does not require considerable resources for data structuring ${ }^{22}$. How these are translated into practice depends on the local circumstances, needs and resources.
Development of international burn registries should facilitate better understanding of burn injury aetiologies in many vulnerable populations. Cooperation between non-governmental organizations, national research networks, national health ministries and local communities to collect and analyse data about specific local aetiologies should increase awareness and knowledge, which could be subsequently applied to preventing burns. These data should include information on number of injuries, at-risk individuals and risk factors to identify vulnerable populations that could be targeted with burn prevention strategies that reflect local culture and availability of resources.

\section{Diagnosis}

Accurate measurement of burn size and depth are central for various treatment interventions. Absence of adequate methods to measure burn depth for superficial partial and deep partial burns makes it difficult to determine which wounds require surgery and which will heal with conservative treatments. New tools have been introduced, many of which are based on laser or thermal imaging technology, to examine skin perfusion to determine burn depth ${ }^{115}$; laser Doppler is a promising example, but others are emerging. Additionally, accurate burn size estimation is particularly challenging in patients with different body shapes, especially those with cachexia or obesity. Several burn measurement tools ${ }^{238}$ are being developed that are provider-independent, with the goal of increased accuracy.

\section{Prognosis}

We also need to develop instruments to evaluate variability among patients, how a patient will respond to burn stress and a patient's likelihood of survival, towards the goal of personalized medicine. For example, sepsis and multiple organ failure are the main causes of mortality in patients after burn injury but burn care providers do not have an accurate means to diagnose, let alone predict, sepsis. Recently, a study compared the ABA sepsis definition with the Mann-Salinas definition and with the Critical Care Sepsis-3 definition ${ }^{239}$ (BOX 1). The study showed that Sepsis-3 is the best and most accurate of the three formulae for identifying sepsis, but was only accurate in $85 \%$ of patients with burn injury ${ }^{239}$. Thus, better definitions need to be developed that more accurately predict and identify sepsis in patients with burn injury.

Sepsis was prospectively predicted with $100 \%$ accuracy during the first surgery using flow cytometry analysis of adipose tissue in a recent study ${ }^{240}$. Although these results are very promising, the study only included 37 patients and this technology cannot easily be translated to every burn centre. However, several other studies are also investigating prediction of sepsis and organ function after burn injury, and it seems only a matter of time until we are able to predict sepsis, multiple organ failure and death in patients. Once we can achieve accurate prediction, the challenge will be to change the trajectory. Some factors are being studied in a holistic approach to predict outcomes in the Glue Grant trial ${ }^{51,52,241}$, a multicentre study examining genomic 
and proteomic changes after burn injury that is producing substantial data. Although the Gluegrant has completed its study, there are still studies and data resulting from this initiative that will enhance our knowledge on personalized medicine.

\section{Acute care}

Aspects of burn care also need further development, including resuscitation ${ }^{242}$. Adequate resuscitation in patients with burn injury is challenging because questions abound regarding volume, composition, formulae and outcome measurement. An emerging technology is the 'burn navigator', which determines resuscitation parameters from urinary output data as a closed loop — adjusting fluid infusion volume automatically based on prior algorithms $\mathrm{s}^{243,244}$. This technology certainly has potential given that organ function such as cardiac function, and liver and kidney perfusion can be integrated, but it does require optimization. For example, urinary output is not ideal as it depends on several variables and can be misleading in determining resuscitation parameters (see above).

Another area of care that requires improvement is wound coverage. To overcome the need for uninjured donor skin in autologous STSG, artificial skin substitutes have been introduced to the market, and include TransCyte, CEA, cultured skin substitutes and various others $^{245,246}$ (TABLE 1). However, their application is limited in patients with burn injury due to cost, labour time and availability. Nonetheless, these products have changed the way we think about tissue-engineered $\operatorname{skin}^{170}$. Currently, various approaches could change the treatment paradigm for burn wound care including stem cells ${ }^{247}$, stem cell 'guns' and 3D printing of autologous cells ${ }^{248}$ to recreate a patient's skin that would reduce the risk of scarring and the need for donor sites, moving closer to our goal of no scar, no pain.

\section{Long-term care}

The historical belief that once burn wounds have healed, the patient is recovered is no longer valid. Indeed, the last phase of burn care - rehabilitation - is becoming one of the most important. Burn survivors have a substantially greater mortality than their matched non-injured counterparts, with the greatest risk of death during the first year after the injury (declining each year thereafter $)^{11,127}$. This increased mortality is related to trauma and mental illness, clearly indicating that survivors of burn injury have considerable care needs that are not being met. Indeed, burn survivors also have a decreased quality of life and struggle for a long time to adjust to their new life ${ }^{210}$. At this time, it is not fully understood how we will diagnose, treat and prevent these longterm morbidities and, therefore, long-term studies to gain better insights are urgently needed.

The response to a burn injury is very complex, and so is the approach to its care. Although we have made some important strides in terms of survival following a burn injury, burn care needs to change over the next decades. New technologies need to be adopted in all phases of the recovery process to further improve burn outcomes, acutely and in the long term.

Published online: 13 February 2020
1. American Burn Association. National Burn Repository 2019 Update, Report of data from 2009-2018 ameriburn.site-ym.com https://ameriburn.site-ym. com/store/ViewProduct.aspx?id=14191872 (2019).

2. Lee, R. C. Injury by electrical forces: pathophysiology, manifestations, and therapy. Curr. Probl. Surg. 34 677-764 (1997).

3. Nguyen, C. M., Chandler, R., Ratanshi, I. \& Logsetty, S. in Handbook of Burns Vol. 1 (eds. Jeschke, M. G., Kamolz, L.-P., Sjöberg, F. \& Wolf, S. E.) 529-547 (Springer, 2020).

4. Jeschke, M. G. et al. Long-term persistance of the pathophysiologic response to severe burn injury. PLoS One 6, e21245 (2011)

This is the first trial to demonstrate that the pathophysiological, immune and inflammatory changes persist for a long time after burn injury. Jeschke, M. G et al. Pathophysiologic response to severe burn injury. Ann. Surg. 248, 387-401 (2008).

6. Stanojcic, M., Abdullahi, A., Rehou, S., Parousis, A. \& Jeschke, M. G. Pathophysiological response to burn injury in adults. Ann. Surg. 267, 576-584 (2018)

7. Porter, C. et al. The metabolic stress response to burn trauma: current understanding and therapies. Lancet 388, 1417-1426 (2016).

8. Burnett, E., Gawaziuk, J. P., Shek, K. \& Logsetty, S. Healthcare resource utilization associated with burns and necrotizing fasciitis. J. Burn Care Res. 38 e886-e891 (2017).

9. Enns, J. et al. Mental and physical health outcomes in parents of children with burn injuries as compared with matched controls. J. Burn Care Res. 37, e18-e26 (2016).

10. Logsetty, S. et al. Mental health outcomes of burn a longitudinal population-based study of adults hospitalized for burns. Burns 42, 738-744 (2016).

11. Mason, S. A. et al. Increased rate of long-term mortality among burn survivors. Ann. Surg. 269 1192-1199 (2019).

Together with Logsetty et al. (2016), this paper looks at long-term outcomes from the mortality and mental health aspects.
12. National Academies of Sciences, Engineering, and Medicine. A National Trauma Care System: Integrating Military and Civilian Trauma Systems to Achieve Zero Preventable Deaths After Injury (National Academies Press, 2016)

13. World Health Organization. Burns. WHO https://www. who.int/en/news-room/fact-sheets/detail/burns (WHO, 2018)

14. Stone, J. et al. Outcomes in adult survivors of childhood burn injuries as compared with matched controls. J. Burn Care Res. 37, e166-e173 (2016).

15. Rybarczyk, M. M. et al. A systematic review of burn injuries in low- and middle-income countries: epidemiology in the WHO-defined African region. Afr. J. Emerg. Med. 7, 30-37 (2017)

16. Stylianou, N., Buchan, I \& Dunn, K. W. A review of the international Burn Injury Database (iBID) for England and Wales: descriptive analysis of burn injuries 2003-2011. BMJ Open 5, e006184 (2015).

17. Sethi, J., Gawaziuk, J. P., Cristall, N. \& Logsetty, S. The relationship between income and burn incidence in Winnipeg, Manitoba, Canada: a population health study. J. Burn Care Res. 39, 645-651 (2018).

18. Padalko, A., Cristall, N., Gawaziuk, J. P. \& Logsetty, S Social complexity and risk for pediatric burn injury: a systematic review. J. Burn Care Res. 40, 478-499 (2019).

19. Peck, M. \& Pressman, M. A. The correlation between burn mortality rates from fire and flame and economic status of countries. Burns 39, 1054-1059 (2013).

20. Spiwak, R., Logsetty, S., Afifi, T. O. \& Sareen, J. Severe partner perpetrated burn: examining a nationally representative sample of women in India. Burns 41, 1847-1854 (2015)

21. Ready, F. L. et al. Epidemiologic shifts for burn injury in Ethiopia from 2001 to 2016: implications for public health measures. Burns 44, 1839-1843 (2018).
22. World Health Organization. Global Burn Registry WHO https://www.who.int/violence injury prevention burns/gbr/en/ (WHO, 2019). A resource that illustrates the creation of a cheap, easy-to-use registry to better understand the epidemiology of burn injuries around the world.

23. Smolle, C. et al. Recent trends in burn epidemiology worldwide: a systematic review. Burns 43, 249-257 (2017).

24. Greenhalgh, D. G. Management of burns. N. Engl. J. Med. 380, 2349-2359 (2019).

25. World Life Expectancy. World Health Rankings https:/ www.worldlifeexpectancy.com/cause-of-death/fires/ by-country (2017).

26. Bayuo, J., Agbenorku, P., Amankwa, R. \& Agbenorku, M Epidemiology and outcomes of burn injury among older adults in a Ghanaian tertiary hospital. Burns Open 2 98-103 (2018).

27. Sanghavi, P., Bhalla, K. \& Das, V. Fire-related deaths in India in 2001: a retrospective analysis of data. Lancet 373, 1282-1288 (2009).

28. Tegtmeyer, L. C. et al. Retrospective analysis on thermal injuries in children-demographic etiological and clinical data of German and Austrian pediatric hospitals 2006-2015-approaching the new German burn registry. Burns 44, 150-157 (2018).

29. Dissanaike, S. \& Rahimi, M. Epidemiology of burn injuries: highlighting cultural and socio-demographic aspects. Int. Rev. Psychiatry 21, 505-511 (2009).

30. Atwell, K., Bartley, C., Cairns, B. \& Charles, A. The effect of pre-existing seizure disorders on mortality and hospital length of stay following burn injury. J. Burn Care Res. 40, 979-982 (2019).

31. Kallinen, O., Maisniemi, K., Böhling, T., Tukiainen, E. \& Koljonen, V. Multiple organ failure as a cause of death in patients with severe burns. J. Burn Care Res. 33, 206-211 (2012).

32. Nielson, C. B., Duethman, N. C., Howard, J. M Moncure, M. \& Wood, J. G. Burns: pathophysiology of systemic complications and current management. J. Burn Care Res. 38, e469-e481 (2017). 
33. Osuka, A., Ogura, H., Ueyama, M., Shimazu, T. \& Lederer, J. A. Immune response to traumatic injury: harmony and discordance of immune system homeostasis. Acute Med. Surg. 1, 63-69 (2014).

34. Sood, R. F. et al. Early leukocyte gene expression associated with age, burn size, and inhalation injury in severely burned adults. J. Trauma Acute Care Surg. 80, 250-257 (2016)

35. Xiao, W. et al. A genomic storm in critically injured humans. J. Exp. Med. 208, 2581-2590 (2011).

36. Rowan, M. P. et al. Burn wound healing and treatment: review and advancements. Crit. Care 19, (2015). This article provides a comprehensive description of cytokine storm after burn and other traumatic injuries.

37. Muthu, K. et al. Perturbed bone marrow monocyte development following burn injury and sepsis promote hyporesponsive monocytes. J. Burn Care Res. 29, 12-21 (2008)

38. Rae, L., Fidler, P. \& Gibran, N. The physiologic basis of burn shock and the need for aggressive fluid resuscitation. Crit. Care Clin. 32, 491-505 (2016).

39. Guillory, A., Clayton, R., Herndon, D. \& Finnerty, C. Cardiovascular dysfunction following burn injury: what we have learned from rat and mouse models. Int. J. Mol. Sci. 17, 53 (2016).

40. Lorente, J. A. et al. Systemic hemodynamics, gastric intramucosal PCO2 changes, and outcome in critically ill burn patients. Crit. Care Med. 28, 1728-1735 (2000).

41. Vaughn, L. \& Beckel, N. Severe burn injury, burn shock, and smoke inhalation injury in small animals. Part 1: burn classification and pathophysiology: severe burn injury and smoke inhalation injury. J. Vet. Emerg. Crit. Care 22, 179-186 (2012).

42. Keck, M., Herndon, D. H., Kamolz, L. P., Frey, M. \& Jeschke, M. G. Pathophysiology of burns. Wien. Med. Wochenschr. 159, 327-336 (2009).

43. Brooks, N. C. et al. XBP- $1 \mathrm{~s}$ is linked to suppressed gluconeogenesis in the ebb phase of burn injury. Mol. Med. 19, 72-78 (2013).

44. Jeschke, M. G. et al. Calcium and ER stress mediate hepatic apoptosis after burn injury. J. Cell. Mol. Med. 13, 1857-1865 (2009).

45. Jeschke, M. G. The hepatic response to thermal injury: is the liver important for postburn outcomes? Mol. Med. 15, 337-351 (2009).

46. Jeschke, M. G. Postburn hypermetabolism: past, present, and future. J. Burn Care Res. 37, 86-96 (2016).

47. Sidossis, L. S. et al. Browning of subcutaneous white adipose tissue in humans after severe adrenergic stress. Cell Metab. 22, 219-227 (2015).

48. Gore, D. C. et al. Hyperglycemia exacerbates muscle protein catabolism in burn-injured patients. Crit. Care Med. 30, 2438-2442 (2002)

49. Hart, D. W. et al. Persistence of muscle catabolism after severe burn. Surgery 128, 312-319 (2000).

50. Patsouris, D. et al. Burn induces browning of the subcutaneous white adipose tissue in mice and humans. Cell Rep. 13, 1538-1544 (2015).

51. Finnerty, C. C. et al. Determination of burn patient outcome by large-scale quantitative discovery proteomics. Crit. Care Med. 41, 1421-1434 (2013)

52. Seok, J. et al. Genomic responses in mouse models poorly mimic human inflammatory diseases. Proc. Natl Acad. Sci. USA 110, 3507-3512 (2013). This landmark study demonstrates that the genomic response observed in humans to burn injury is highly correlated with that to trauma and simulated sepsis, suggesting that if an intervention targeting the response is effective for one type of insult, it may be effective against all three.

53. Singer, M. et al. The Third International Consensus Definitions for Sepsis and Septic Shock (Sepsis-3). JAMA 315, 801-810 (2016)

54. Kupper, T. S., Green, D. R., Durum, S. K. \& Baker, C. C. Defective antigen presentation to a cloned $T$ helper cell by macrophages from burned mice can be restored with interleukin-1. Surgery 98, 199-206 (1985).

55. Miyazaki, H., Kinoshita, M., Ono, S., Seki, S. \& Saitoh, D. Burn-evoked reactive oxygen species immediately after injury are crucial to restore the neutrophil function against postburn infection in mice. Shock 44, 252-257 (2015)

56. Hampson, P. et al. Neutrophil dysfunction, immature granulocytes, and cell-free DNA are early biomarkers of sepsis in burn-injured patients: a prospective observational cohort study. Ann. Surg. 265 1241-1249 (2017).

57. Antonacci, A. C. et al. Autologous and allogeneic mixed-lymphocyte responses following thermal injury in man: the immunomodulatory effects of interleukin 1 , interleukin 2, and a prostaglandin inhibitor. WY-18251. Clin. Immunol. Immunopathol. 30, 304-320 (1984)

58. Baker, C. C., Miller, C. L. \& Trunkey, D. D. Predicting fatal sepsis in burn patients. J. Trauma 19, 641-648 (1979).

59. Murphy, T. J., Paterson, H. M., Mannick, J. A. \& Lederer, J. A. Injury, sepsis, and the regulation of Toll-like receptor responses. J. Leukoc. Biol. 75 400-407 (2003).

60. Schlüter, B., König, W., Köller, M., Erbs, G. \& Müller, F. E. Differential regulation of $\mathrm{T}$ - and B-lymphocyte activation in severely burned patients. J. Trauma 31, 239-246 (1991).

61. Wood, J. J. et al. Inadequate interleukin 2 production. Ann. Surg. 200, 311-320 (1984)

62. Messingham, K. A., Faunce, D. E. \& Kovacs, E. J. Alcohol, injury, and cellular immunity. Alcohol 28 137-149 (2002)

63. Lachiewicz, A. M., Hauck, C. G., Weber, D. J., Cairns, B. A \& van Duin, D. Bacterial infections after burn injuries: impact of multidrug resistance. Clin. Infect. Dis. 65, 2130-2136 (2017)

64. Rech, M. A. et al. Outcomes in burn-injured patients who develop sepsis. J. Burn Care Res. 40, 269-273 (2019).

65. Ballard, J. et al. Positive fungal cultures in burn patients: a multicenter review. J. Burn Care Res. 29 213-221 (2008)

66. Gomez, R. et al. Causes of mortality by autopsy findings of combat casualties and civilian patients admitted to a burn unit. J. Am. Coll. Surg. 208 348-354 (2009).

67. Horvath, E. E. et al. Fungal wound infection (not colonization) is independently associated with mortality in burn patients. Ann. Surg. 245, 978-985 (2007).

68. Gosain, A. \& Gamelli, R. L. Role of the gastrointestina tract in burn sepsis. J. Burn Care Rehabil. 26, 85-91 (2005).

69. Magnotti, L. J., Xu, D. Z., Lu, Q. \& Deitch, E. A Gut-derived mesenteric lymph: a link between burn and lung injury. Arch. Surg. 134, 1333-1340 (1999).

70. Herndon, D. N. \& Zeigler, S. T. Bacterial translocation after thermal injury. Crit. Care Med. 21, S50-S54 (1993).

71. Baron, P. et al. Gut failure and translocation following burn and sepsis. J. Surg. Res. 57, 197-204 (1994).

72. Deitch, E. A. \& Berg, R. Bacterial translocation from the gut. J. Burn Care Rehabil. 8, 475-482 (1987).

73. Beckmann, N., Pugh, A. M. \& Caldwell, C. C. Burn injury alters the intestinal microbiome's taxonomic composition and functional gene expression. PLoS One 13, e0205307 (2018).

74. Earley, Z. M. et al. Burn injury alters the intestinal microbiome and increases gut permeability and bacterial translocation. PLoS One 10, e0129996 (2015).

This article discusses changes in the gut microbiota in human patients with burn injuries and in a mouse model of burn injury.

75. Deitch, E. A. Maejima, K. \& Berg R. Effect of oral antibiotics and bacterial overgrowth on the translocation of the $\mathrm{GI}$ tract microflora in burned rats. J. Trauma 25, 385-392 (1985)

76. Deitch, E. A., Lu, Q., Feketeova, E., Hauser, C. J. \& $\mathrm{Xu}, \mathrm{D}$.-Z. Intestinal bacterial overgrowth induces the production of biologically active intestinal lymph. J. Trauma 56, 105-110 (2004).

77. Diamant, M., Blaak, E. E. \& de Vos, W. M. Do nutrientgut-microbiota interactions play a role in human obesity, insulin resistance and type 2 diabetes? Obes. Rev. 12, 272-281 (2010)

78. Mutlu, E. et al. Intestinal dysbiosis: a possible mechanism of alcohol-induced endotoxemia and alcoholic steatohepatitis in rats. Alcohol. Clin. Exp. Res. 33, 1836-1846 (2009).

79. Sekirov, I., Russell, S. L., Antunes, L. C. M. \& Finlay, B. B. Gut microbiota in health and disease. Physiol. Rev. 90, 859-904 (2010)

80. Shimizu, K. et al. Altered gut flora and environment in patients with severe SIRS. J. Trauma 60, 126-133 (2006).

81. Rehberg, S. et al. Pathophysiology, management and treatment of smoke inhalation injury. Expert. Rev. Respir. Med. 3, 283-297 (2009).

82. Endorf, F. W. \& Gamelli, R. L. Inhalation injury, pulmonary perturbations, and fluid resuscitation. J. Burn Care Res. 28, 80-83 (2007).

83. Grigorian, A. et al. Rising mortality in patients with combined burn and trauma. Burns 44, 1989-1996 (2018).
84. Krishnan, P., Frew, Q., Green, A., Martin, R. \& Dziewulski, P. Cause of death and correlation with autopsy findings in burns patients. Burns 39 583-588 (2013)

85. Capek, K. D., Culnan, D. M., Merkley, K. Huan, T. T. \& Trocme, S. in Total Burn Care 5th edn (ed. Herndon, D. N.) 435-444 (Elsevier, 2018).

86. American Burn Association. Prevention. ameriburn.org https://ameriburn.org/prevention (2019).

87. American Burn Association. Verification Criteria Effective October 1, 2019. ameriburn.org http:// ameriburn.org/quality-care/verification/verificationcriteria/verification-criteria-effective-october-1-2019/ (2019)

88. Peck, M., Molnar, J. \& Swart, D. A global plan for burn prevention and care. Bull. World Health Organ. 87, 802-803 (2009)

89. World Health Organization. Burn prevention: success stories, lessons learned https://apps.who.int/iris/ bitstream/handle/10665/97938/9789241501187. eng.pdf (WHO, 2011)

90. Folz, D . H \& Shults, C. The impact of state fire safe cigarette policies on fire fatalities, injuries, and incidents. J. Emerg. Manag. 15, 379-389 (2017).

91. Laing, R. M. \& Bryant, V. Prevention of burn injuries to children involving nightwear. N. Z. Med. J. 104 363-365 (1991).

92. Harvey, L. A., Connolley, S. \& Harvey, J. G. Clothingrelated burns in New South Wales, Australia: impact of legislation on a continuing problem. Burns 41, 58-64 (2015)

93. Erdmann, T. C., Feldman, K. W., Rivara, F. P., Heimbach, D. M. \& Wall, H. A. Tap water burn prevention: the effect of legislation. Pediatrics $\mathbf{8 8}$, 572-577 (1991)

94. Haddon, W. The changing approach to the epidemiology, prevention, and amelioration of trauma: the transition to approaches etiologically rather than descriptively based. Inj. Prev. 5, 231-235 (1999).

95. Peck, M. D. et al. Burns and injuries from non-electric appliance fires in low- and middle-income countries. Burns 34, 312-319 (2008).

96. Sadeghi-Bazargani, H. et al. Exploring possible causes of fatal burns in 2007 using Haddon's Matrix: a qualitative study. J. Inj. Violence Res. 7, 1-6 (2015)

97. Kahn, S. A., Patel, J. H., Lentz, C. W. \& Bell, D. E. Firefighter burn injuries: predictable patterns influenced by turnout gear. J. Burn Care Res. 33, 152-156 (2012).

98. Burgess, J., Watt, K., Kimble, R. M. \& Cameron, C. M Combining technology and research to prevent scald injuries (the Cool Runnings intervention): randomized controlled trial. J. Med. Internet Res. 20, e 10361 (2018)

99. Parbhoo, A., Louw, Q. A. \& Grimmer-Somers, K. Burn prevention programs for children in developing countries require urgent attention: a targeted literature review. Burns 36, 164-175 (2010).

100. Jin, R., Wu, P., Ho, J. K., Wang, X. \& Han, C. Five-year epidemiology of liquefied petroleum gas-related burns. Burns 44, 210-217 (2018).

101. Teven, C. M. \& Gottlieb, L. J. The four-quadrant approach to ethical issues in burn care. AMA J. Ethics 20, 595-601 (2018).

102. Mohammad, A., Branicki, F. \& Abu-Zidan, F. M Educational and clinical impact of Advanced Trauma Life Support (ATLS) courses: a systematic review. World J. Surg. 38, 322-329 (2013).

103. Breederveld, R. S., Nieuwenhuis, M. K. Tuinebreijer, W. E. \& Aardenburg, B. Effect of training in the emergency management of severe burns on the knowledge and performance of emergency care workers as measured by an online simulated burn incident. Burns 37, 281-287 (2011).

104. Kearns, R. D. et al. Advanced burn life support for day-to-day burn injury management and disaster preparedness: stakeholder experiences and student perceptions following 56 advance burn life support crimes. J. Burn Care Res. 36, 455-464 (2015)

105. Klein, M. B. et al. Geographic access to burn center hospitals. JAMA 302, 1774-1781 (2009). This examination of geographical distribution of regional burn care highlights the variation in access to verified burn centres by ground and rotary air transport across the USA, with $\sim 80 \%$ of the US population being within 2 hours from a verified burn centre, illustrating the potential for limited resources in a disaster

106. Henry, S. ATLS 10th edition offers new insights into managing trauma patients. Bulletin of the American College of Sugeons http://bulletin.facs.org/2018/06 atls-10th-edition-offers-new-insights-into-managingtrauma-patients/ (2018). 
107. Lund C. \& Browder, N. The estimation of areas of burns. Surg. Gynecol. Obstet. 79, 352-358 (1944).

108. Wallace, A. B. The exposure treatment of burns Lancet 257, 501-504 (1951).

109. ISBI Practice Guidelines Committee. ISBI practice guidelines for burn care. Burns 42, 953-1021 (2016). This major consensus project establishes guidelines for global burn care, including resource-limited environments.

110. Foster, K. N. \& Holmes, J. H. Inhalation injury: state of the science 2016. J. Burn Care Res. 38, 137-141 (2017).

111. Ching, J. A. et al. An analysis of inhalation injury diagnostic methods and patient outcomes. J. Burn Care Res. 37, e27-e32 (2016).

112. Williams, J. F. et al. Comparison of traditional burn wound mapping with a computerized program. J. Burn Care Res. 34, e29-e35 (2013)

113. Benjamin, N. C. et al. Accuracy of currently used paper burn diagram vs a three-dimensional computerized model. J. Burn Care Res. 38, e254-e260 (2017).

114. Burke-Smith, A., Collier, J. \& Jones, I. A comparison of non-invasive imaging modalities: infrared thermography, spectrophotometric intracutaneous analysis and laser Doppler imaging for the assessment of adult burns. Burns 41, 1695-1707 (2015).

115. Wearn, C. et al. Prospective comparative evaluation study of laser Doppler imaging and thermal imaging in the assessment of burn depth. Burns 44, 124-133 (2018).

116. Sen, C. K., Ghatak, S., Gnyawali, S. C., Roy, S. \& Gordillo, G. M. Cutaneous imaging technologies in acute burn and chronic wound care. Plast. Reconstr. Surg. 138, 119S-128S (2016)

117. Ganapathy, P. et al. Dual-imaging system for burn depth diagnosis. Burns 40, 67-81 (2014).

118. Burmeister, D. M. et al. Noninvasive techniques for the determination of burn severity in real time. J. Burn Care Res. 38, e180-e191 (2017)

119. Greenhalgh, D. G. Burn resuscitation: the results of the ISBI/ABA survey, Burns 36, 176-182 (2010).

120. Chung, K. K. et al. Simple derivation of the initial fluid rate for the resuscitation of severely burned adult combat casualties: in silico validation of the rule of 10 . J. Trauma 69, S49-S54 (2010)

121. Mosier, M. J. et al. Early acute kidney injury predicts progressive renal dysfunction and higher mortality in severely burned adults. J. Burn Care Res. 31, 83-92 (2010).

122. Kumar, A. B. et al. Fluid resuscitation mediates the association between inhalational burn injury and acute kidney injury in the major burn population. J. Crit. Care 38, 62-67 (2017).

123. Sine, C. R. et al. Acute respiratory distress syndrome in burn patients. J. Burn Care Res. 37, e461-e469 (2016).

124. Cochran, A. Inhalation injury and endotracheal intubation. J. Burn Care Res. 30, 190-191 (2009).

125. Amtmann, D. et al. Satisfaction with life over time in people with burn injury: a National Institute on Disability, Independent Living, and Rehabilitation Research burn model system study. Arch. Phys. Med. Rehabil. 101, S63-S70 (2020).

126. Wiechman, S. A. et al. Reasons for distress among burn survivors at 6,12 , and 24 months postdischarge: a burn injury model system investigation. Arch. Phys. Med. Rehabil. 99 1311-1317 (2018).

127. Mason, S. A. et al. Association between burn injury and mental illness among burn survivors: a population-based, self-matched, longitudinal cohort study. J. Am. Coll. Surg. 225, 516-524 (2017).

128. Davê, D. R., Nagarjan, N., Canner, J. K., Kushner, A. L. \& Stewart, B. T. Rethinking burns for low \& middle-income countries: differing patterns of burn epidemiology, care seeking behavior, and outcomes across four countries. Burns 44, 1228-1234 (2018).

129. Young, A. W. et al. Guideline for burn care under austere conditions. J. Burn Care Res. 38, e497-e509 (2017).

130. Jeng, J., Gibran, N. \& Peck, M. Burn care in disaster and other austere settings. Surg. Clin. North. Am. 94 893-907 (2014)

131. American College of Surgeons. Advanced Trauma Life Support. facs.org https://www.facs.org/quality $\% 20$ programs/trauma/atls (2019)

132. American Burn Association. Burn Center Referral Criteria. ameriburn.org http://ameriburn.org/wp-content/ uploads/2017/05/burncenterreferralcriteria.pdf (2006).

133. Roberts, G. et al. The Baux score is dead. Long live the Baux score: a 27-year retrospective cohort study of mortality at a regional burns service. J. Trauma. Acute Care Surg. 72, 251-256 (2012)

134. Klein, M. B. et al. Benchmarking outcomes in the critically injured burn patient. Ann. Surg. 259, 833-841 (2014).

This paper establishes benchmarks as goals for burn outcomes based on $>500$ patients treated using standard operating procedures and prospectively documented in a centralized database.

135. Romanowski, K. et al. The frailty tipping point: determining which patients are targets for intervention in a burn population. Burns 45 1051-1056 (2019).

136. Rehou, S., Shahrokhi, S., Thai, J., Stanojcic, M. \& Jeschke, M. G. Acute phase response in critically ill elderly burn patients. Crit. Care Med. 47, 201-209 (2019).

137. Grigorian, A. et al. 23 burns in octogenarians: 80 is the new 60. J. Burn Care Res. 40, S19 (2019).

138. Lawrence, A. et al. Colloid administration normalizes resuscitation ratio and ameliorates "fluid creep".

J. Burn Care Res. 31, 40-47 (2010).

139. Ivy, M. E. et al. Intra-abdominal hypertension and abdominal compartment syndrome in burn patients. J. Trauma 49, 387-391 (2000).

140. O'Mara, M. S., Slater, H., Goldfarb, I. W. \& Caushaj, P. F. A prospective, randomized evaluation of intraabdominal pressures with crystalloid and colloid resuscitation in burn patients. J. Trauma $\mathbf{5 8}$ 1011-1018 (2005)

141. Torres, L. N., Chung, K. K., Salgado, C. L., Dubick, M. A \& Torres Filho, I. P. Low-volume resuscitation with normal saline is associated with microvascular endothelial dysfunction after hemorrhage in rats, compared to colloids and balanced crystalloids. Crit. Care 21, 160 (2017)

142. MacLennan, S. \& Williamson, L. M. Risks of fresh frozen plasma and platelets. J. Trauma 60, S46-S50 (2006).

143. Rizzo, J. A., Rowan, M. P., Driscoll, I. R., Chung, K. K $\&$ Friedman, B. C. Vitamin C in burn resuscitation. Crit. Care Clin. 32, 539-546 (2016).

144. Dubick, M. A., Williams, C., Elgjo, G. I. \& Kramer, G. C. High-dose vitamin $C$ infusion reduces fluid requirements in the resuscitation of burn-injured sheep. Shock 24, 139-144 (2005).

145. Tanaka, H. et al. Reduction of resuscitation fluid volumes in severely burned patients using ascorbic acid administration: a randomized, prospective study. Arch. Surg. 135, 326-331 (2000).

146. Fowler, A. A. et al. Effect of vitamin C infusion on organ failure and biomarkers of inflammation and vascular injury in patients with sepsis and severe acute respiratory failure: the CITRIS-ALI randomized clinical trial. JAMA 322, 1261-1270 (2019).

147. Kahn, S. A., Beers, R. J. \& Lentz, C. W. Resuscitation after severe burn injury using high-dose ascorbic acid a retrospective review. J. Burn Care Res. 32, 110-117 (2011).

148. Buehner, M. et al. Oxalate nephropathy after continuous infusion of high-dose vitamin $\mathrm{C}$ as an adjunct to burn resuscitation. J. Burn Care Res. 37 e374-e379 (2016)

149. Sartor, Z., Kesey, J. \& Dissanaike, S. The effects of intravenous vitamin $C$ on point-of-care glucose monitoring. J. Burn Care Res. 36, 50-56 (2015)

150. Neff, L. P. Allman, J. M. \& Holmes, J. H. The use of theraputic plasma exchange (TPE) in the setting of refractory burn shock. Burns 36, 372-378 (2010)

151. Klein, M. B. et al. The beneficial effects of plasma exchange after severe burn injury. J. Burn Care Res. 30, 243-248 (2009)

152. Heering, P. et al. Cytokine removal and cardiovascular hemodynamics in septic patients with continuous venovenous hemofiltration. Intensive Care Med. 23 288-296 (1997)

153. Payen, D et al. Impact of continuous venovenous hemofiltration on organ failure during the early phase of severe sepsis: a randomized controlled trial. Crit. Care Med. 37, 803-810 (2009).

154. Chung, K. K. et al. High-volume hemofiltration in adult burn patients with septic shock and acute kidney injury: a multicenter randomized controlled trial. Crit. Care 21, 289 (2017)

155. You, B. et al. Early application of continuous highvolume haemofiltration can reduce sepsis and improve the prognosis of patients with severe burns. Crit. Care 22, 173 (2018).

156. Pruitt, B. A. Jr, O'Neill, J. A. Jr, Moncrief, J. A. \& Lindberg, R. B. Successful control of burn-wound sepsis. JAMA 203, 1054-1056 (1968).
157. Burke, J. F., Bondoc, C. C. \& Quinby, W. C. Primary burn excision and immediate grafting: a method shortening illness. J. Trauma 14, 389-395 (1974).

158. Desai, M. H. et al. Early burn wound excision significantly reduces blood loss. Ann. Surg. 211, 753-762 (1990)

159. Herndon, D N et al. A comparison of conservative versus early excision. Therapies in severely burned patients. Ann. Surg. 209, 547-553 (1989).

160. Hermans, M. H. E. Results of an internet survey on the treatment of partial thickness burns, full thickness burns, and donor sites. J. Burn Care Res. 28 835-847 (2007)

161. Carta, T. et al. Properties of an ideal burn dressing: a survey of burn survivors and front-line burn healthcare providers. Burns 45, 364-368 (2019).

162. Horch, R., Stark, G. B., Kopp, J. \& Spilker, G. Cologne Burn Centre experiences with glycerol-preserved allogeneic skin: part I: clinical experiences and histological findings (overgraft and sandwich technique). Burns 20, S23-S26 (1994).

163. Ren, J. et al. The use of noncultured regenerative epithelial suspension for improving skin color and scars: a report of 8 cases and review of the literature. J. Cosmet. Dermatol. 18, 1487-1494 (2019).

164. Merchant, N. et al. Management of adult patients with buttock and perineal burns: the Ross Tilley Burn Centre experience. J. Trauma. Acute Care Surg. 77, 640-648 (2014).

165. Murphy, P. S. \& Evans, G. R. D. Advances in wound healing: a review of current wound healing products. Plast. Surg. Int. 2012, 190436 (2012).

166. Chocarro-Wrona, C., López-Ruiz, E., Perán, M Gálvez-Martín, P. \& Marchal, J. A. Therapeutic strategies for skin regeneration based on biomedical substitutes. J. Eur. Acad. Dermatol. Venereol. 33 484-496 (2019)

167. Xue, M., Zhao, R., Lin, H. \& Jackson, C. Delivery systems of current biologicals for the treatment of chronic cutaneous wounds and severe burns. Adv. Drug. Deliv. Rev. 129, 219-241 (2018).

168. Chouhan, D. Dey, N., Bhardwaj, N. \& Mandal, B. B. Emerging and innovative approaches for wound healing and skin regeneration: current status and advances. Biomaterials 216, 119267 (2019).

169. Nicholas, M. N., Jeschke, M. G. \& Amini-Nik, S. Methodologies in creating skin substitutes. Cell. Mol. Life Sci. 73, 3453-3472 (2016).

170. Sheikholeslam, M., Wright, M. E. E., Jeschke, M. G. \& Amini-Nik, S. Biomaterials for skin substitutes. Adv. Healthc. Mater. 7, 1700897 (2018)

171. PolarityTE. SkinTE for providers. polarityte.com https://www. polarityte.com/products/skinTE-providers (2020).

172. Shevchenko, R. V., James, S. L. \& James, S. E. A review of tissue-engineered skin bioconstructs available for skin reconstruction. J. R. Soc. Interface 7, 229-258 (2010).

173. Davison-Kotler, E., Sharma, V., Kang, N. V. \& Garcia-Gareta E. A universal classification system of skin substitutes inspired by factorial design. Tissue Eng. Part B Rev. 24, 279-288 (2018).

174. Nicholas, M. N. \& Yeung, J. Current status and future of skin substitutes for chronic wound healing. J. Cutan Med. Surg. 21, 23-30 (2017)

175. Halim, A. S., Khoo, T. L. \& Yussof, S. J. M. Biologic and synthetic skin substitutes: an overview. Indian. J. Plast. Surg. 43, S23-S28 (2010).

176. Mohan, R., Bajaj, A. \& Gundappa, M. Human amnion membrane: potential applications in oral and periodontal field. J. Int. Soc. Prev. Community Dent. 7 15-21 (2017)

177. van Zuijlen, P. P. M. et al. Tissue engineering in burn scar reconstruction. Burns Trauma 3, 18 (2015).

178. Haddad, A. G., Giatsidis, G., Orgill, D. P. \& Halvorson, E. G. Skin substitutes and bioscaffolds. Clin. Plast. Surg. 44, 627-634 (2017).

179. Fang, T., Lineaweaver, W. C., Sailes, F. C., Kisner, C. \& Zhang, F. Clinical application of cultured epithelial autografts on acellular dermal matrices in the treatment of extended burn injuries. Ann. Plast. Surg. 73, 509-515 (2014)

180. Gauglitz, G G Korting H C Pavicic, T Ruzicka, T. $\&$ Jeschke, M. G. Hypertrophic scarring and keloids: pathomechanisms and current and emerging treatment strategies. Mol. Med. 17, 113-125 (2011).

181. Finnerty, C. C. et al. Hypertrophic scarring: the greatest unmet challenge after burn injury. Lancet 388, 1427-1436 (2016)

182. Vincent, A. S. et al. Human skin keloid fibroblasts display bioenergetics of cancer cells. J. Invest. Dermatol. 128, 702-709 (2008). 
183. Tsai, C.-H. \& Ogawa, R. Keloid research: current status and future directions. Scars Burn. Heal. 5 , 2059513119868659 (2019).

184. Nitzschke, S. L. et al. Wound healing trajectories in burn patients and their impact on mortality. J. Burn Care Res. 35, 474-479 (2014).

185. Lester, M. E., Hazelton, J., Dewey, W. S., Casey, J. C. $\&$ Richard, R. Influence of upper extremity positioning on pain, paresthesia, and tolerance. J. Burn Care Res. 34, e342-e350 (2013).

186. Nedelec, B., Serghiou, M. A., Niszczak, J., McMahon, M $\&$ Healey, T. Practice guidelines for early ambulation of burn survivors after lower extremity grafts. J. Burn Care Res. 33, 319-329 (2012)

187. Richard, R. \& Santos-Lozada, A. R. Burn patient acuity demographics, scar contractures, and rehabilitation treatment time related to patient outcomes. J. Burn Care Res. 38, 230-242 (2017)

188. Wiechman, S. A. \& Patterson, D. R. ABC of burns. Psychosocial aspects of burn injuries. BMJ 329 391-393 (2004)

189. Ravenek, M. J., Skarakis-Doyle, E Spaulding, S. J. Jenkins, M. E. \& Doyle, P. C. Enhancing the conceptual clarity and utility of the international classification of functioning, disability $\&$ health: the potential of a new graphic representation. Disabil. Rehabil. 35 , 1015-1025 (2013)

190. Miller, T. et al. Quality-of-life loss of people admitted to burn centers, United States. Qual. Life Res. 22, 2293-2305 (2013)

191. Öster, C., Willebrand, M., Dyster-Aas, J., Kildal, M. \& Ekselius, L. Validation of the EQ-5D questionnaire in burn injured adults. Burns 35, 723-732 (2009).

192. Kildal, M., Andersson, G., Fugl-Meyer, A. R. Lannerstam, K. \& Gerdin, B. Development of a brief version of the Burn Specific Health Scale (BSHS-B). J. Trauma 51, 740-746 (2001).

193. Spronk, I. et al. Health related quality of life in adults after burn injuries: a systematic review. PLoS One 13, e0197507 (2018).

194. Griffiths, C. et al. A systematic review of patient reported outcome measures (PROMs) used in child and adolescent burn research. Burns 41, 212-224 (2015).

195. Griffiths, C. et al. A systematic review of patientreported outcome measures used in adult burn research. J. Burn Care Res. 38, e521-e545 (2017).

196. Tyack, Z et al. Measuring the impact of burn scarring on health-related quality of life: development and preliminary content validation of the Brisbane Burn Scar Impact Profile (BBSIP) for children and adults. Burns 41, 1405-1419 (2015)

197. Kool, M. B. Geenen, R Egberts, M. R Wanders, H. $\&$ Van Loey, N. E. Patients' perspectives on quality of life after burn. Burns 43, 747-756 (2017).

198. Meirte, J. et al. Classification of quality of life subscales within the ICF framework in burn research: identifying overlaps and gaps. Burns 40, 1353-1359 (2014).

199. Meirte, J. et al. Convergent and discriminant validity of quality of life measures used in burn populations. Burns 43, 84-92 (2017).

200. Edgar, D., Dawson, A., Hankey, G., Phillips, M. \& Wood, F. Demonstration of the validity of the SF-36 for measurement of the temporal recovery of quality of life outcomes in burns survivors. Burns 36 1013-1020 (2010)

201. Tyack, Z., Kimble, R., McPhail, S., Plaza, A. \& Simons, M Psychometric properties of the Brisbane Burn Scar Impact Profile in adults with burn scars. PLoS One 12, e0184452 (2017).

202. Griffiths, C. et al. The development and validation of the CARe burn scale-adult form: a Patient-Reported Outcome Measure (PROM) to assess quality of life for adults living with a burn injury. J. Burn Care Res. 40, 312-326 (2019)

203. Kazis, L. E. et al. Development of the life impact burn recovery evaluation (LIBRE) profile: assessing burn survivors' social participation. Qual. Life Res. 26 2851-2866 (2017)

204. McMahon, H. A., Ndem, I., Gampper, L., Gampper, T. J. $\&$ DeGeorge, B. R. Quantifying burn injury-related disability and quality of life in the developing world: a primer for patient-centered resource allocation. Ann. Plast. Surg. 82, S433-S436 (2019).

205. Kazis, L. E. et al. Methods for assessment of health outcomes in children with burn injury: the multi-center benchmarking study. J. Trauma. Acute Care Surg. 73, S179-S188 (2012).

206. Ryan, C. M. et al. Benchmarks for multidimensional recovery after burn injury in young adults: the development, validation, and testing of the American Burn Association/Shriners Hospitals for Children young adult burn outcome questionnaire. J. Burn Care Res. 34, e121-e142 (2013)

207. Kazis, L. E. et al. Recovery curves for pediatric burn survivors: advances in patient-oriented outcomes. JAMA Pediatr. 170, 534 (2016).

This paper shows considerable deficits in paediatric burn survivors and provides recovery curves based on the Burn Outcomes Questionnaire, which can be used as benchmark for the expected recovery after burn injury in children

208. Spronk, I. et al. Recovery of health-related quality of life after burn injuries: an individual participant data meta-analysis. PLoS One 15, e0226653 (2020). This paper shows the long-term outcomes in adult burn survivors and presents recovery curves based on SF-36 and EQ-5D data, which can be used in clinical practice to inform patients and professionals on expected HROOL outcomes after burn injuries in adults.

209. Wasiak, J. et al. Female patients display poorer burnspecific quality of life 12 months after a burn injury. Injury 48, 87-93 (2017).

210. Chin, T. L. et al. Trends 10 years after burn injury: a burn model system national database study. Burns 44, 1882-1886 (2018).

211. Levi, B. et al. The associations of gender with social participation of burn survivors: a life impact burn recovery evaluation profile study. J. Burn Care Res. 39, 915-922 (2018)

212. Spronk, I. et al. Predictors of health-related quality of life after burn injuries: a systematic review. Crit. Care 22, 160 (2018).

213. Spronk, I. et al. Health related quality of life 5-7 years after minor and severe burn injuries: a multicentre cross-sectional study. Burns 45, 1291-1299 (2019).

214. Öster, C., Willebrand, M. \& Ekselius, L. Health-related quality of life 2 years to 7 years after burn injury. J. Trauma 71, 1435-1441 (2011).

215. Needham, D. M. et al. Improving long-term outcomes after discharge from intensive care unit: report from a stakeholders' conference. Crit. Care Med. 40 502-509 (2012)

216. Deeter, L. et al. Hospital-acquired complications alter quality of life in adult burn survivors: report from a burn model system. Burns 45, 42-47 (2019).

217. Yoder, L. H., McFall, D. C. \& Glaser, D. N. Quality of life of burn survivors treated in the military burn center. Nurs. Outlook 65, S81-S89 (2017).

218. Al Ghriwati, N. et al. Two-year gender differences in satisfaction with appearance after burn injury and prediction of five-year depression: a latent growth curve approach. Arch. Phys. Med. Rehabil. 98 2274-2279 (2017).

219. Sinha, l. et al. Head and neck burns are associated with long-term patient-reported dissatisfaction with appearance: a burn model system national database study. Burns 45, 293-302 (2019).

220. Gerrard, P. et al. Validation of the community integration questionnaire in the adult burn injury population. Qual. Life Res. 24, 2651-2655 (2015)

221. Ryan, C. M., Cartwright, S., Schneider, J. C., Tompkins, R. G. \& Kazis, L. E. The burn outcome questionnaires: patient and family reported outcome metrics for children of all ages. Burns 42, 1144-1145 (2016).

222. World Health Organization. International Classification of Functioning, Disability and Health (ICF). https:/ www.who.int/classifications/icf/en/ (WHO, 2018).

223. Osborne, C. L. et al. The multicenter benchmarking study of burn injury: a content analysis of the outcome measures using the international classification of functioning, disability and health. Burns $\mathbf{4 2}$ 1396-1403 (2016).

224. Spronk, I., Legemate, C. M., Polinder, S. \& v an Baar, M. E. Health-related quality of life in children after burn injuries. J. Trauma. Acute Care Surg. 85, 1110-1118 (2018)

225. Pan, R. et al. Health-related quality of life in adolescent survivors of burns: agreement on selfreported and mothers' and fathers' perspectives. Burns 41, 1107-1113 (2015).

226. Meyer, W. J. et al. Adolescent survivors of burn injuries and their parents' perceptions of recovery outcomes: do they agree or disagree? J. Trauma. Acute Care Surg. 73, S213-S220 (2012).

227. Mason, S. T. et al. Return to work after burn injury: a systematic review. J. Burn Care Res. 33, 101-109 (2012).

228. Quinn, T., Wasiak, J. \& Cleland, H. An examination of factors that affect return to work following burns: a systematic review of the literature. Burns 36 , 1021-1026 (2010)
229. Goei, H. et al. Return to work after specialised burn care: a two-year prospective follow-up study of the prevalence, predictors and related costs. Injury 47, 1975-1982 (2016).

230. Christiansen, M. et al. Time to school re-entry after burn injury is quite short. J. Burn Care Res. 28, 478-481 (2007).

231. Pan, R. et al. School reintegration of pediatric burn survivors: an integrative literature review. Burns $\mathbf{4 4}$ 494-511 (2018)

232. Mason, S. A et al. Burn center care reduces acute health care utilization after discharge: a populationbased analysis of 1,895 survivors of major burn injury. Surgery 162, 891-900 (2017).

233. Duke, J. M. et al. Increased admissions for musculoskeletal diseases after burns sustained during childhood and adolescence. Burns 41, 1674-1682 (2015).

234. Randall, S. M. et al. Long-term musculoskeletal morbidity after adult burn injury: a population-based cohort study. BMJ Open 5, e009395 (2015).

235. Polychronopoulou, E., Herndon, D. N. \& Porter, C. The long-term impact of severe burn trauma on musculoskeletal health. J. Burn Care Res. 39, 869-880 (2018).

236. Duke, J. M. et al. Diabetes mellitus after injury in burn and non-burned patients: a population based retrospective cohort study. Burns 44, 566-572 (2018)

237. Duke, J. M., Rea, S., Boyd, J. H., Randall, S. M. \& Wood, F. M. Mortality after burn injury in children: a 33-year population-based study. Pediatrics 135 , e903-e910 (2015).

238. Parvizi, D. et al. BurnCase 3D software validation study: burn size measurement accuracy and inter-rater reliability. Burns 42, 329-335 (2016).

239. Yan, J. et al. Sepsis criteria versus clinical diagnosis of sepsis in burn patients: a validation of current sepsis scores. Surgery 164, 1241-1245 (2018).

240. Chen, P., Stanojcic, M. \& Jeschke, M. G. Septic predictor index: a novel platform to identify thermally injured patients susceptible to sepsis. Surgery 163, 409-414 (2018)

241. Finnerty, C. C. et al. Proteomics improves the prediction of burns mortality: results from regression spline modeling. Clin. Transl. Sci. 5, 243-249 (2012)

242. Mason, S. A. et al. Hold the pendulum: rates of acute kidney injury are increased in patients who receive resuscitation volumes less than predicted by the Parkland equation. Ann. Surg. 264, 1142-1147 (2016).

243. Cancio, L. C., Salinas, J. \& Kramer, G. C. Protocolized resuscitation of burn patients. Crit. Care Clin. 32, 599-610 (2016)

244. Serio-Melvin, M. L. et al. Burn shock and resuscitation. J. Burn Care Res. 38, e423-e431 (2017).

245. Jeschke, M. G., Finnerty, C. C., Shahrokhi, S. Branski, L. K. \& Dibildox, M. Wound coverage technologies in burn care. J. Burn Care Res. 34, 612-620 (2013)

246. Jeschke, M. G. et al. Wound coverage technologies in burn care: established techniques. J. Burn Care Res. 39, 313-318 (2018)

247. Amini-Nik, S. et al. Stem cells derived from burned skin - the future of burn care. EBioMedicine 37, 509-520 (2018)

248. Hakimi, N. et al. Handheld skin printer: in situ formation of planar biomaterials and tissues. Lab Chip 18, 1440-1451 (2018)

249. Brannen, A. L. et al. A randomized prospective trial of hyperbaric oxygen in a referral burn center population. Am. Surg. 63, 205-208 (1997).

250. de Durante, G. et al. ARDSNet lower tidal volume ventilatory strategy may generate intrinsic positive end-expiratory pressure in patients with acute respiratory distress syndrome. Am. J. Respir. Crit. Care Med. 165, 1271-1274 (2002).

251. Kearns, R. D. et al. Guidelines for burn care under austere conditions: introduction to burn disaster airway and ventilator management, and fluid resuscitation. J. Burn Care Res. 37, e427-e439 (2016).

252. Cancio, L. C. et al. Guidelines for burn care under austere conditions: surgical and nonsurgical wound management. J. Burn Care Res. 38, 203-214 (2017)

253. Wetta-Hall, R., Jost, J. C., Jost, G., Praheswari, Y. $\&$ Berg-Copas, G. M. Preparing for burn disasters: evaluation of a continuing education training course for pre-hospital and hospital professionals in Kansas. J. Burn Care Res. 28, 97-104 (2007)

254. Spiwak, R., Lett, R., Rwanyuma, L. \& Logsetty, S. Examining perception and actual knowledge change among learners in a standardized burn course. Plast. Surg. 23, 221-224 (2015). 
255. Spiwak, R., Lett, R., Rwanyuma, L. \& Logsetty, S. Creation of a standardized burn course for low income countries: meeting local needs. Burns $\mathbf{4 0}$, 1292-1299 (2014)

256. Interburns. Improving Quality in Burn Care. interburns.org https://interburns.org/training/ (2019).

257. Peck, M., Jeng, J. \& Moghazy, A. Burn resuscitation in the austere environment. Crit. Care Clin. 32, 561-565 (2016)

258. Burmeister, D. M. et al. Operational advantages of enteral resuscitation following burn injury in resourcepoor environments: palatability of commercially available solutions. J. Spec. Oper. Med. 19, 76-81 (2019).

259. Gómez, B. I. et al. Enteral resuscitation with oral rehydration solution to reduce acute kidney injury in burn victims: evidence from a porcine model. PLoS One 13, e0195615 (2018)

260. Georgiadis, J., Nascimento, V. B., Donat, C., Okereke, I. \& Shoja, M. M. Dakin's solution: "One of the most important and far-reaching contributions to the armamentarium of the surgeons". Burns 45 1509-1517 (2019)

261. Hirsch, T. et al. Antimicrobial activity of clinically used antiseptics and wound irrigating agents in combination with wound dressings. Plast. Reconstr. Surg. $127,1539-1545$ (2011).

262. US Fire Administration. Choosing and using fire extinguishers. usfa.fema.gov https://www.usfa.fema. gov/prevention/outreach/extinguishers.html (2017).

263. Szczesny, B. et al. Time-dependent and organ-specific changes in mitochondrial function, mitochondrial DNA integrity, oxidative stress and mononuclear cell infiltration in a mouse model of burn injury. PLoS One 10, e0143730 (2015)

264. Choudhry, M. A., Gamelli, R. L. \& Chaudry, I. H in Yearbook of Intensive Care and Emergency Medicine 2004 (ed. Vincent, J.-L.) 16-26 (Springer, 2004).

265. Rae, L. et al. Differences in resuscitation in morbidly obese burn patients may contribute to high mortality. J. Burn Care Res. 34, 507-514 (2013).

266. Rossiter, N. D., Chapman, P. \& Haywood, I. A. How big is a hand? Burns 22, 230-231 (1996)

267. Jean, J. Bioengineered skin: the self-assembly approach. J. Tissue Sci. Eng. https://doi.org/ 10.4172/2157-7552.S5-001 (2013).

268. Nathoo, R., Howe, N. \& Cohen, G. Skin substitutes: an overview of the key players in wound management J. Clin. Aesthetic Dermatol. 7, 44-48 (2014).

269. Sheridan, R. Closure of the excised burn wound: autografts, semipermanent skin substitutes, and permanent skin substitutes. Clin. Plast. Surg. 36, 643-651 (2009)

270. Takami, Y., Yamaguchi, R., Ono, S. \& Hyakusoku, H. Clinical application and histological properties of autologous tissue-engineered skin equivalents using an acellular dermal matrix. J. Nippon. Med. Sch. 81, 356-363 (2014)

271. Holmes IV, J. H. et al. A comparative study of the ReCell ${ }^{\circledast}$ device and autologous split-thickness meshed skin graft in the treatment of acute burn injuries. J. Burn Care Res. 39, 694-702 (2018).

272. Gravante, G. et al. A randomized trial comparing ReCell system of epidermal cells delivery versus classic skin grafts for the treatment of deep partial thickness burns. Burns 33, 966-972 (2007).

273. Moustafa, M. et al. Randomized, controlled, singleblind study on use of autologous keratinocytes on a transfer dressing to treat nonhealing diabetic ulcers. Regen. Med. 2, 887-902 (2007).

274. Hernon, C. A. et al. Clinical experience using cultured epithelial autografts leads to an alternative methodology for transferring skin cells from the laboratory to the patient. Regen. Med. 1, 809-821 (2006).

275. Gerlach, J. C. et al. Autologous skin cell spraytransplantation for a deep dermal burn patient in an ambulant treatment room setting. Burns 37 e19-e23 (2011).

276. Lee, H. Outcomes of sprayed cultured epithelial autografts for full-thickness wounds: a single-centre experience. Burns 38, 931-936 (2012).

277. Romanelli, M. Dini, V., Bertone M. Barbanera, S. \& Brilli, C. OASIS wound matrix versus Hyaloskin in the treatment of difficult-to-heal wounds of mixed arterial/ venous aetiology. Int. Wound J. 4, 3-7 (2007)

278. Hodde, J. P., Ernst, D. M. J. \& Hiles, M. C. An investigation of the long-term bioactivity of endogenous growth factor in OASIS Wound Matrix. J. Wound Care 14, 23-25 (2005).

279. Philandrianos, C. et al. Comparison of five dermal substitutes in full-thickness skin wound healing in a porcine model. Burns 38, 820-829 (2012).

280. Kogan, S., Halsey, J. \& Agag, R. L. Biologics in acute burn injury. Ann. Plast. Surg. 83, 26-33 (2019).

281. Kim, J. S., Kaminsky, A. J., Summitt, J. B. \& Thayer, W. P. New innovations for deep partial-thickness burn treatment with ACell Matristem Matrix. Adv. Wound Care 5, 546-552 (2016).

282. Yannas, I. V. \& Burke, J. F. Design of an artificial skin. I. Basic design principles. J. Biomed. Mater. Res. 14, 65-81 (1980)
283. Hicks, K. E., Huynh, M. N., Jeschke, M. \& Malic, C. Dermal regenerative matrix use in burn patients: a systematic review. J. Plast. Reconstr. Aesthetic Surg. 72, 1741-1751 (2019)

284. Wainwright, D. J. Use of an acellular allograft dermal matrix (AlloDerm) in the management of full-thickness burns. Burns 21, 243-248 (1995).

285. Debels, H., Hamdi, M., Abberton, K. \& Morrison, W. Dermal matrices and bioengineered skin substitutes: a critical review of current options. Plast. Reconstr. Surg. Glob. Open 3, e284 (2015).

286. Boa, O. et al. Prospective study on the treatment of lower-extremity chronic venous and mixed ulcers using tissue-engineered skin substitute made by the self-assembly approach. Adv. Skin. Wound Care 26, 400-409 (2013).

287. Zelen, C. M. et al. A prospective, randomised, controlled, multi-centre comparative effectiveness study of healing using dehydrated human amnion/chorion membrane allograft, bioengineered skin substitute or standard of care for treatment of chronic lower extremity diabetic ulcers. Int. Wound J. 12, 724-732 (2015).

288. Boyce, S. T. et al. Cultured skin substitutes reduce donor skin harvesting for closure of excised, full-thickness burns. Ann. Surg. 235, 269-279 (2002).

\section{Acknowledgements}

M.G.J. acknowledges support from the US National Institute of General Medical Sciences (R01 GM087285), Canadian Institutes of Health Research (\#123336) and CFI Leader's Opportunity Fund (Project \#25407). M.A.C. acknowledges support from the US National Institute of General Medical Sciences (R01GM128242). N.S.G. acknowledges support from US National Institute for Disability Independent Living and Rehabilitation Research.

\section{Author contributions}

Introduction (M.G.J.); Epidemiology (S.L.); Mechanisms/ pathophysiology (M.A.C., K.K.C. and M.G.J.); Diagnosis, screening and prevention (N.S.G. and S.L.); Management (K.K.C.); Quality of life (M.E.v.B. and N.S.G.); Outlook (M.G.J.); Overview of the Primer (M.G.J.). With the exception of M.G.J., all authors are listed alphabetically.

\section{Competing interests}

The authors declare no competing interests.

Publisher's note

Springer Nature remains neutral with regard to jurisdictional claims in published maps and institutional affiliations.

C) Springer Nature Limited 2020 